\title{
La nascita e l'evoluzione della cultura epigrafica in Magna Grecia: documenti, temi, sfide e prospettive
}

\section{The birth and the evolution of the epigraphic culture in Magna Graecia: documents, themes, challenges and perspectives}

\author{
Giovanni Boffa \\ Università del Salento \\ giovanni.boffa@unisalento.it
}

Riassunto: Lo scopo del presente lavoro è quello di illustrare la nascita e i primi sviluppi della cultura epigrafica nel contesto della Magna Grecia. Di tali complessi e articolati fenomeni, che risultano essere parte integrante di quello più ampio della nascita e della prima diffusione dell'alfabeto greco, si cercherà di cogliere le radici e le implicazioni sociali, attraverso l'esame di documenti idonei a far emergere ambiti, contesti, occasioni, mezzi, modi e pratiche collegate all'uso della scrittura.

Parole chiave: Cultura epigrafica. Nascita dellalfabeto greco. Magna Grecia. Aspetti e implicazioni sociali della scrittura.

Abstract: This study aims to illustrate the rise and the early developments of the epigraphic culture in Magna Graecia. It will pay special attention to the social roots, aspects and implications of these two highly complex phenomena, which are involved in the wider events of the rise and the early evolution of the Greek alphabet. For this purpose, the work will take into examination some meaningful documents, in order to highlight, occasions, contexts, means, ways and practices related to the use of writing.

Keywords: Epigraphic culture. Early Greek alphabet and literacy. Magna Graecia Social aspects and implications of writing.

Recepción: 23.01.2020 | Aceptación: 09.04.2020 


\section{Premessa: la genesi di una cultura epigrafica come fenomeno sociale}

Uno di tratti distintivi e qualificanti del Progetto AELAW. Ancient European Languages and Writings risiede non solo nella definizione delloggetto della ricerca (le lingue e le scritture antiche d'Europa) e negli ambiziosi obiettivi (la costruzione di una rete di specialisti in grado di sviluppare una banca dati dei documenti attestanti le suddette lingue e scritture $)^{1}$ ma anche nell'approccio alla materia stessa, che possiede un taglio marcatamente sociale, ossia rispondente alla precisa volontà di analizzare i fenomeni linguistici e scrittori osservandoli all'interno dei differenti e concreti contesti sociali nei quali essi si manifestano di volta in volta e come parte integrante degli stessi. Questo indirizzo è stato ben presente nella tavola rotonda intitolata "La nascita delle antiche culture epigrafiche europee. Grecia, Italia, Iberia (secoli VIII-VI a. C.)", tenutasi nell'ambito del Convegno conclusivo del Progetto, ${ }^{2}$ all'interno della quale sono stato invitato a tenere la relazione che costituisce la base del presente contributo. ${ }^{3}$ Luso dellespressione "culture epigrafiche", infatti, una scelta che condivido in pieno, appare assai denso di significati in relazione alla sopra nominata intenzione. "Culture epigrafiche" sottintende non solo la presenza, in un determinato contesto sociale, di un sistema di scrittura ma anche il suo impiego all'interno di un arco cronologico (è, dunque, insita, nell'espressione, una dimensione diacronica), ampio tanto da permettere l'interiorizzazione, la sedimentazione di tale elemento (che va pensato come un sistema, vale dire come un particolare insieme di conoscenze teorico-pratiche quali la sequenza dei segni, la loro forma, i loro valori fonetici, il modo di eseguirli, considerando anche i possibili e differenti supporti impiegati, le caratteristiche delle superfici scrittorie, i sistemi d'impaginazione, gli strumenti

1 Cito dal sito web E-COST. European Cooperation in Science and Technology (<cost. eu/actions/IS1407>): "The main objective of the Action is the co-ordination of researchers dedicated to the study of the different ancient languages and writings with the aim of creating an ample work team capable of establishing the foundations for the creation, for the first time, of a large online data bank which will permit the cataloguing of all the currently known documents in this type of languages, thus introducing this important part of the European cultural heritage into the $21^{\text {st }}$ century".

2 Mi riferisco al Convegno Internazionale "Lingue e scritture paleoeuropee: sfide e prospettive degli studi”, EEHAR. Escuela Española de Historia y Arqueología en Roma, 13-15 marzo 2019.

3 Colgo qui l'occasione per ringraziare il Comitato scientifico e organizzatore dell'evento e Enrico Benelli, Coordinatore della suddetta tavola rotonda. 
idonei alla sua 'messa in opera', siano essi professionali, come gli stili e gli scalpelli, o, per così dire, improvvisati ed estemporanei $)^{4}$ e il suo conseguente divenire, appunto, "cultura", ossia parte integrante e sostanziale del più ampio e generale sistema di saperi, costumi e comportamenti della società che se ne serve, con numerose e peculiari ricadute di ordine teorico e pratico su vari aspetti del suo sistema simbolico, della sua vita e delle sue attività. ${ }^{5}$ L'approccio sociologico allo studio dei sistemi di scrittura, con particolare riferimento al mondo antico e in relazione ai delicati problemi che attengono ai come e ai perché della loro genesi e diffusione, ha, comè noto radici ben salde e importanti nel lungo, assai variegato e produttivo filone di studi in cui l'attenzione più o meno marcata alla dimensione antropologica e sociale della scrittura è assurta al ruolo di chiave di lettura primaria dei documenti osservati. Penso, ovviamente, ai contributi, che potremmo definire fondamentali, di H. Innis, ${ }^{6}$ J. Goody e I. Watt, ${ }^{7}$ D. De Kerckove, ${ }^{8}$ sul piano dell'impostazione generale; a W. J. Ong, ${ }^{9}$ R. Finnegan, ${ }^{10}$ E. Havelock, ${ }^{11}$ J. Assmann,,${ }^{12}$ R. Thomas, ${ }^{13}$ D. Olson, con particolare riferimento al tema del rapporto fra oralità e scrittura e, nel caso di Olson, alla psicologia cognitiva; ${ }^{14}$ a H.-I. Marrou e K. Robb in rapporto

4 Su questi aspetti mi limito a segnalare, in una bibliografia vasta e variegata, gli approcci di Cardona 1981; Pandolfini e Prosdocimi 1990; Ong 2002; De Kerckhove 2008; Pappas 2011.

5 Sul concetto di cultura, sulla sua definizione e il suo sviluppo in ambito sociologico vid., per un orientamento, De Biasi 2002; Griswold 2005; Sciolla 2006.

$6 \quad$ Innis 1986.

7 Goody 1986; Goody e Watt 1968.

8 De Kerckhove 2008.

9 Ong 2002.

10 Finnegan 1988.

11 Havelock 1963; 1982; 1986.

12 Assman 2011.

13 Thomas 1989; 1992.

14 Olson 2006; Olson e Torrance 1991; 2001; 2009; Olson, Torrance e Hildyard 1985; Taylor e Olson 1995. Si tratta di un settore in cui si sono conseguiti risultati scientifici molto interessanti in relazione ai meccanismi fisiologici, psichici e psicologici collegati all'apprendimento e all'uso della scrittura, che, tuttavia, ancora non sono stati adeguatamente recepiti e valorizzati in ambito epigrafico ma che, a mio avviso, sono potenzialmente forieri di interessanti spunti ermeneutici in grado di spiegare in maniera più sofisticata, rispetto a quanto accade attualmente, alcuni fenomeni scrittori. Un esempio calzante in tal senso viene da un recente e assai suggestivo lavoro di D. De Kerckhove, che ha suggerito un collegamento fra il mutamento nella direzione della scrittura operato dai greci, rispetto al modello fenicio, e i fattori neurofisiologici legati alla codifica e alla decodifica dei segni alfabetici (De Kerckhove 2008, 63-82). 
agli studi sull'educazione nel mondo antico; ${ }^{15}$ al già citato G. R. Cardona per il raffinato taglio antropologico della sua ricerca; ${ }^{16}$ a F. Coulmas, sul versante della sociolinguistica. ${ }^{17}$

"Culture epigrafiche", in definitiva, è un'espressione che pone la scrittura all'intersezione di discipline diverse quali l'epigrafia e la sociologia. Essa, tuttavia, è questo un secondo punto fondamentale da considerare, non fa riferimento alla scrittura tout court ma ad un suo particolare e definito campo di applicazione, ad una sua peculiare dimensione pratica, quella epigrafica, che è connotata dall'utilizzo, come superficie scrittoria, di supporti non deperibili, ossia di unamplissima gamma di oggetti diversi per forma, dimensioni e funzioni, il che trova numerose e importanti implicazioni sia sul piano dell'esecuzione (le tecniche e gli strumenti con i quali si scrive) ${ }^{18}$ sia su quello dei contesti e della finalità comunicative di ciò che si scrive. Alla luce di quanto detto, il tema di questo lavoro, dunque, "la nascita e l'evoluzione della cultura epigrafica in Magna Grecia”, sarà sviluppato attraverso l'illustrazione di una rassegna di documenti idonei a far emergere ambiti, contesti, occasioni, mezzi, modi e pratiche collegate all'uso della scrittura in ambito magnogreco, a mettere in luce le implicazioni sociali dello stesso e i fattori in gioco nella sua apparizione.

\section{Gli esordi della cultura epigrafica in Magna Grecia: documenti e contesti significativi}

La genesi della cultura epigrafica nei centri magnogreci è un problema di elevata complessità, in virtù del fatto che, è questo un punto importante e qualificante, tale processo è, in realtà, parte integrante del più ampio e generale fenomeno che riguarda la nascita dell'alfabeto greco stesso, i suoi primi usi,

15 Marrou 1964; Robb 1994. Vid. anche la panoramica in Johnson 2015.

16 Cardona 1981. Sulla scia di Cardona ha lavorato, in tempi recenti, G. M. Facchetti 2009. Sull'antropologia della scrittura, sulla sua evoluzione e sulle recenti tendenze nel panorama internazionale, in riferimento non solo al mondo antico, vid. Barton e Papen 2010; Bartlett et al. 2011.

17 Coulmas 2002; 2013.

18 Non pare inopportuno sottolineare il fatto che, con particolare riferimento all'VIII secolo a.C., fase in cui l'uso della scrittura epigrafica è essenzialmente ristretto all'ambito privato, la quasi totalità dei documenti epigrafici vada riferita non alla mano di 'professionisti', quali ceramisti, scalpellini e incisori, la cui attività dipende di una comitenza ed è (con particolare riguardo ai secondi e ai terzi) è legata agli usi pubblici della scrittura, ma a quella di soggetti che scrivono per sé e di propria mano, solitamente usando la tecnica del graffito. Su usi, tecniche, strumenti e materiali vid. Guarducci 1967, 429-468. 
la sua diffusione all'interno dell'orizzonte culturale greco e, cosa non trascurabile, anche la sua precoce trasmissione a contesti culturali allogeni, con particolare riferimento all'ambiente italico. Il documento che esemplifica perfettamente questo stato di cose, trovando attinenza con la totalità delle questioni appena enumerate, è la ben nota e discussa iscrizione più antica dalla necropoli di Osteria dell'Osa (fig. 1). ${ }^{19}$

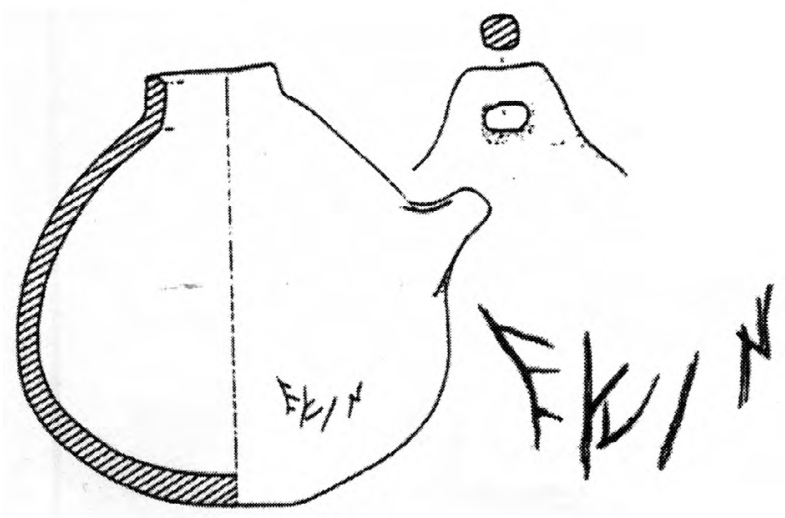

Fig. 1. Roma, necropoli laziale di località Osteria dell'Osa, 770 a.C. Fiasca d'impasto monoansata di produzione locale, recante iscrizione graffita: disegno dell'oggetto e fac-simile dellepigrafe (da Bietti Sestieri 1992a, tav. 20, n. 16).

Questa straordinaria epigrafe, forse la più antica iscrizione greca conosciuta, viene a costituire, infatti, un elemento di intersezione fra tre differenti scritture: l'alfabeto greco, quello etrusco e quello latino. Ciò avviene non solo in forza delle caratteristiche che attengono al luogo e al contesto di rinvenimento, alla scrittura impiegata e alla datazione, ma anche in virtù delle differenti e contrastanti ipotesi di lettura delle quali essa è stata fatta oggetto. Comè noto, infatti, l'iscrizione dell'Osa è stata interpretata come un testo greco (con almeno quattro letture e sei significati differenti), ${ }^{20}$

\section{SEG XLII, 899.}

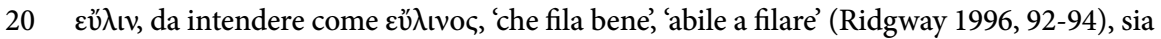
in senso letterale, con riferimento alla produzione tessile, sia in senso metaforico, con riferimento al 'filo' del destino umano (Biondi 1998, 392); عü $\pi \varepsilon \pi \lambda$ oc 'dal bel peplo' (Ampolo 1997); un antroponimo assimilabile, per costruzione, ai noti Eucheir ed Eugrammos, i nomi di due degli artigiani (il terzo era, comè noto, Diopos) portati da Demarato a Tarquinia (Plin., HN 35, 43, 152); عv̉oĩv, "un allotropo di củoî", il grido attribuito a Dioniso infante, che era pronunciato contestualmente alle cerimonie e ai riti legati alla divinità (Peruzzi 1997, 21-22; 1998; Biondi 1998); víkn, interpretando il vasetto che funge da supporto come un premio per una competizione "forse anche di tipo scolastico o letterario" (Canali De Rossi 2005, 168); củvív, nel significato di 'tomba', con riferimento alla sepoltura che conteneva l'oggetto; $\varepsilon u ́ \lambda ı c$, 'ben levigato', in riferimento al vaso (Boffa 2015). 
come un testo in latino ${ }^{21}$ e come una "pseudo-inscription". ${ }^{22}$ Qualunque sia la giusta soluzione, ${ }^{23}$ siamo evidentemente di fronte ad un alfabeto di matrice euboica, lo riconosciamo dal lambda calcidese in particolare, che viene utilizzato su un oggetto personale, un piccolo vaso a fiasco monoansato di pregevole fattura, ${ }^{24}$ verosimilmente appartenuto a una donna, rinvenuto in una non banale 'doppia sepoltura' maschile e femminile, ${ }^{25}$ cronologicamente non più recente del 770 a.C. ${ }^{26}$ e situata all'interno della necropoli di un insediamento laziale, identificabile con l'antica Gabii. ${ }^{27}$

21 Ni lue, che corrisponderebbe a ne luas, un invito a non sciogliere il vaso dalla corda alla quale doveva essere sospeso e, dunque, un invito a non rubarlo (Colonna 2005, 481), oppure a non versarne il contenuto (Bellelli e Benelli 2018, 27).

22 Ovvero un tentativo di scrittura da parte di un illetterato che ha riprodotto a caso alcune lettere (Sass 2005, 155-156).

23 Su questo punto sia consentito rimandare alla discussione in Boffa 2015.

24 Le caratteristiche tipologiche del vaso e la presenza di un foro intenzionale nella sua parte alta sono elementi che hanno fortemente condizionato la discussione sull'interpretazione dell'epigrafie. Su questi aspetti rimando, per una trattazione complessiva a Boffa 2015.

25 La sepoltura a incinerazione n. 482, costituita da un dolio contenente i resti combusti di una donna e quattro vasi più piccoli, fra i quali quello con l'iscrizione fu realizzata all'interno della sepoltura a fossa $\mathrm{n}$. 483 , contenente i resti inumati di un individuo di sesso maschile di circa trent'anni, prima della chiusura della stessa, spostandone il defunto in posizione semi-seduta e appoggiandone la schiena al riempimento di terra creato per sostenere il suddetto dolio (Bietti Sestieri 1992b, 121-126).

26 La sepoltura 482 si situa nel momento finale della fase IIB laziale che, secondo la cronologia cosiddetta tradizionale dell'età del ferro, si estende dall' 830 a.C. al 770 a.C. circa (Bietti Sestieri 1992a, 537). Secondo Ridgway la datazione dell'iscrizione andrebbe fissata, al più tardi, al 775 a.C. (Ridgway 1996, 91). Va ricordato che la suddetta cronologia tradizionale è stata in tempi recenti messa in discussione dai risultati di un ambizioso progetto di ricerca portato avanti dell'Università di Groningen (Nijboer e Van der Plicht 2008; Van der Plicht, Bruins e Nijboer 2009). Gli studiosi coinvolti, tramite l'esame del radiocarbonio di campioni materiali provenienti da una ampia e mirata selezione di siti, che spazia dall'oriente alloccidente mediterraneo, hanno messo a punto una diversa griglia cronologica, all'interno della quale le datazioni convenzionali risultano più alte di almeno 20-50 anni, il che porterebbe a collocare l'iscrizione nel momento finale del IX secolo a.C. Le forti e in larga parte fondate perplessità sollevate sulla validità di tali conclusioni (vid. Fantalkin, Finkelstein e Piasetzky 2011) consigliano, tuttavia, almeno per ora di far riferimento alla cronologia tradizionale.

27 Per un inquadramento della necropoli dell'Osa, che ha restituito seicento tombe a incinerazione e a inumazione riferibili a tutto il corso delletà del ferro laziale (dunque al periodo compreso fra il IX e il VII secolo a.C.), e dell'area di Gabii vid. Bietti Sestieri 1992a, 1992b. Ė appena il caso di ricordare il noto e discusso passaggio di Dionigi di Alicarnasso (D.H. I, 84, 5), nel quale si racconta della paideia di Romolo e Remo, che

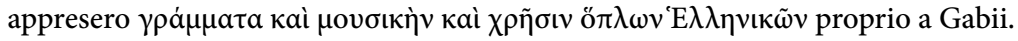


L'iscrizione dell'Osa, ${ }^{28}$ questo è un punto molto importante al netto delle questioni legate al suo significato, evidentemente presuppone l'avvenuto dispiegamento di due fenomeni, di cui essa è allo stesso tempo testimone e risultato: la creazione dell'alfabeto greco, forse in Eubea nel corso della seconda metà del IX secolo a.C., comè già stato suggerito, ${ }^{29} \mathrm{e}$ la sua circolazione anche in contesti non greci, nella fattispecie italici. Quest'ultima va interpretata come epifenomeno delle proiezioni greche dirette verso l'Occidente, e il Tirreno in particolare, risalenti agli inizi dell'VIII secolo a.C., e del contestuale formarsi dell'ambiente coloniale euboico campano, con riferimento privilegiato a Pitecusa (piuttosto che a Cuma), ${ }^{30}$ ovvero a quello che la documentazione consente di individuare come il luogo d'esordio e come il più antico 'motore' e diffusore della prassi e della cultura epigrafica magnogreca, luogo i cui contatti con gli orizzonti etrusco e laziale sono precoci e ben documentati, ${ }^{31}$ anche sul piano epigrafico, come sarà più avanti illustrato con alcuni esempi significativi.

La suddetta cultura si sostanzia, nella sua fase più antica, essenzialmente di documenti di carattere privato, testi in genere brevi e il più delle volte graffiti, meno spesso dipinti, su ceramica atti solitamente ad esprimere il possesso del supporto, la categoria più consistente nell'ambito dell'VIII secolo a.C., oppure indicazioni sul suo contenuto, con riferimento particolare ai graffiti su anfora, $\mathrm{o}$, ancora, la paternità della sua manifattura, ovvero la firma d'artista. ${ }^{32}$

28 Ad essa va forse affiancata la purtroppo mutila ma altrettanto notevole iscrizione retrograda $\alpha \lambda[---$, tracciata su un ossuario biconico rinvenuto nella tomba 21 della necropoli villanoviana Benacci-Caprara di Bologna e inquadrabile nella prima metà del secolo VIII a. C., la cui pertinenza linguistica non è purtroppo individuabile con sicurezza. La prima lettera di tale documento è un eccezionale alpha adagiato; la seconda, mutila, è stata interpretata come chi (Ridgway 1996, 95 n. 50) oppure come lambda (Colonna 2005, 481).

29 Vid. Powell 1991, Ruijgh 1998 e la recente e assai puntuale disamina in Janko 2017.

30 La nascita di Pitecusa, secondo una opinione ormai condivisa, risale al 770 a.C. circa, quella di Cuma, invece, può essere collocata nell'arco del terzo quarto dell'VIII secolo a. C., secondo i risultati di recenti e fruttuose indagini ( $v i d$. Bartoloni e Nizzo 2005, 423 424; D’Agostino 2008; D’Agostino e D’Acunto 2008). L'iscrizione dell'Osa, va sottolineato, risulta pressoché coeva alla nascita di Pitecusa, il che ha delle ricadute significative in chiave storica. Il documento, infatti, stando così le cose, va collocato nel contesto di contatti fra l'orizzonte euboico e quello laziale avviati immediatamente dopo la fondazione o contestualmente alla stessa, se non addirittura prima, nella fase di approccio alla fondazione dell'insediamento (su queste dinamiche e sul più generale approccio di matrice greca al Tirreno agli inizi del fenomeno apecistico vid. Delpino 1997; D’Agostino 1999, 2006), ad opera degli Eubei dei centri metropolitani di Calcide ed Eretria.

31 Buchner 1966; Ridgway 1984, 136-169; D’Agostino 1994.

32 Buchner e Bartoňek 1995 n. 43. 
Liscrizione, purtroppo frammentaria, $\mu \mu \alpha<\lambda>\lambda$ ovo (oppure $\mu \iota \mu a เ o[v o \varsigma$ ) $\grave{\varepsilon} \mu$ í, risalente al 740 a.C. ca., che risulta, pertanto, essere la più antica epigrafe rinvenuta a Pitecusa, graffita con direzione retrograda su anfora grezza di produzione non locale (fig. 2). ${ }^{33}$ La sequenza ugualmente retrograda $\tau \varepsilon \lambda \varepsilon$, risalente alla fine dell'VIII secolo a.C. e graffita su anfora di produzione locale ${ }^{34}$ (fig. 3), e la ben nota iscrizione, sempre retrograda, ]ıvoৎ $\mu^{\prime}$ '̇ं cratere geometrico di produzione locale risalente alla fine dell'VIII secolo a.C. (fig. 4), ${ }^{35}$ ben esemplificano le tre categorie richiamate.

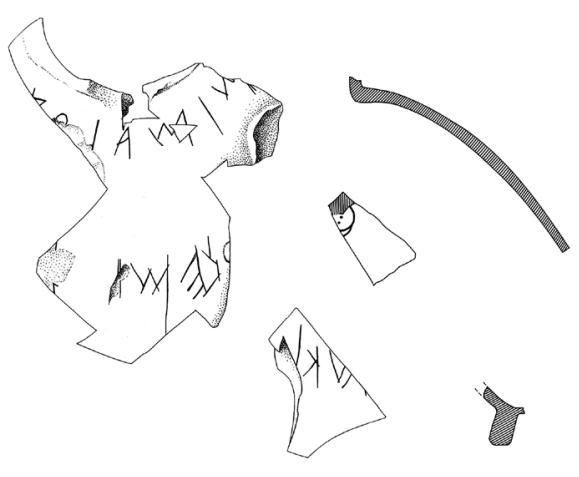

Fig. 2. Ischia, necropoli di S. Montano. Anfora da trasporto di produzione non locale recante iscrizione di possesso graffita. 740 a.C. ca. (da Buchner, Bartoněk 1995, 165, fig. 23).

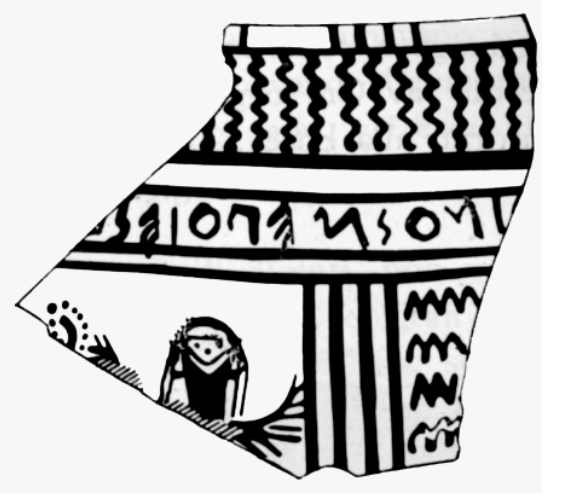

Fig. 4. Cratere geometrico di produzione locale recante firma dell'artista dipinta. Fine dell'VIII secolo a.C. (da Buchner e Bartoněk 1995, 177, fig. 43).

33 SEG XXVIII, 796; Buchner e Bartoňek 1995, 165, n. 23.

34 Buchner e Bartoňek 1995 n. 25. Il termine è di non facile interpretazione. Presumendo che le due vocali siano lunghe (dunque, leggendo $\tau \dot{\eta} \lambda \eta$ ), una sua equivalenza con $\tau \tilde{\eta} \lambda ı \varsigma$, il cosiddetto fieno greco (vid. LSJ s. v.), sembra, dato anche il tipo di supporto, la migliore delle proposte finora avanzate.

35 SEG XLII, 920; Buchner e Bartoněk 1995, 177, nr. B1. 
Tuttavia, il documento che più di ogni altro connota la cultura epigrafica euboico-coloniale più antica è l'iscrizione della cosiddetta coppa di Nestore (fig. 5) ${ }^{36}$ rivenuta nella sepoltura di un fanciullo di circa dodici anni appartenente a quella che è stata definita da Buchner e Ridgway come la "classe media" pitecusana. Si tratta, comè noto, di un testo in metri, un trimetro giambico e due esametri, ${ }^{37}$ che allude ad un passaggio dell'XI libro nell'Iliade, nel quale il re di Pilo Nestore, di ritorno dalla battaglia, beve insieme a Macaone il kykeion preparato dalla schiava Ecamede in una coppa d'oro massiccio. ${ }^{38}$ L'iscrizione pitecusana sembra voler istituire un paragone fra quest'ultimo aggetto e il ben più modesto vaso di fabbricazione rodia sul quale essa e graffita, vaso che, dal canto suo, vanta lo speciale potere di far nascere, in colui che da esso beve,

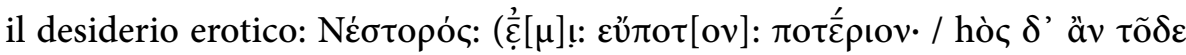

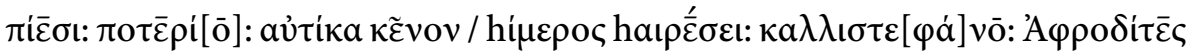
("Di Nestore io sono la coppa, da cui si beve bene, e chi beva da questa coppa, subito lui prenderà il desiderio di Afrodite dalla bella corona"). ${ }^{39}$

\section{Buchner e Bartoňek 1995 n. 44}

37 Su questo punto vid. Pavese 1996, 9-10.

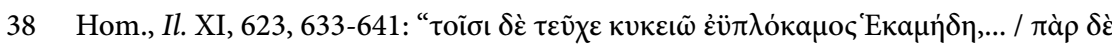

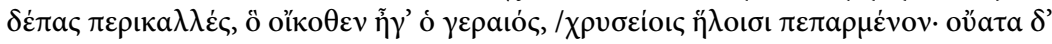

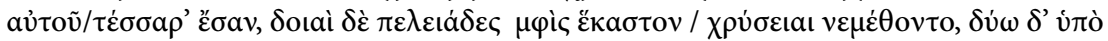

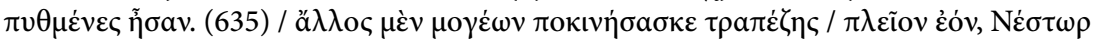

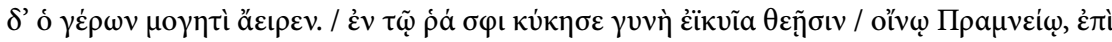

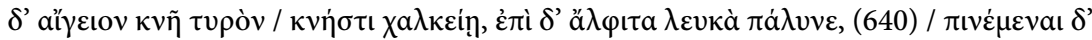

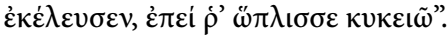

39 Il testo e la traduzione sono quelli proposti in Valerio 2017. Va detto, che la discussione sul significato complessivo dell'iscrizione della coppa di Nestore e sulla sua intenzione comunicativa, risulta molto ampia e articolata e numerose sono le proposte formulate su tali questioni nel corso degli anni, che appaiono sostanzialmente polarizzate: da un lato vi è chi ritiene che, in realtà, il testo pitecusano non intenda fare riferimenti all'lliade e che il Nestore della coppa, dunque, sia un abitante reale di Pitecusa (il che implica interpretare il primo rigo come una mera iscrizione di possesso); dall'altro, e si tratta dei più, chi pensa, con varie sfumature, l'esatto opposto. Per una sintesi critica sulle varie proposte interpretative, segnalo Buchner e Bartoněk 1995, 146-154, nr. A1; Pavese 1996 (fra i pochi che propendono per l'assenza di riferimenti all'epica); Valerio 2017. Assai suggestivo Faraone 1996, che interpreta il testo della seconda e della terza linea assimilandolo a quello di una maledizione. Non pare inopportuno ricordare, infine, come l'interpretazione del documento sia in parte condizionata anche dal modo con il quale si decide di integrare la lacuna al primo rigo, punto sul quale si sono esercitati in molti. Alla luce della nuova e accurata misurazione della sua estensione fatta da C. O. Pavese (1996), che in buona sostanza individua spazio per sole tre lettere, la soluzione oggi più ampiamente condivisa sembra essere rappresentata dalla prima persona singolare del verbo essere, $\grave{\varepsilon} \mu$ í, nel dialetto euboico (Arena 1994, 119), che risulta la più naturale, anche in virtù dei non banali confronti rinvenibili all'interno dell'epigrafia pitecusana stessa (Valerio 2017); tuttavia, l'integrazione proposta illo tempore da M. Guarducci 
L'epigrafe, per il suo tenore generale, sembra rimandare al consumo del vino e alla presenza delle etere nel contesto del simposio. ${ }^{40} \mathrm{Si}$ tratta di elementi non comuni nel panorama generale dell'epigrafia magnogreca, che ritroviamo, verosimilmente, nella più tarda ma ancora arcaica iscrizione tarantina di Melusa (fig. 6), graffita sotto il piede di una bella kylix a figura nere databile fra il 540 e il 520 a.C., decorata con tre scene di battaglia e una scena che

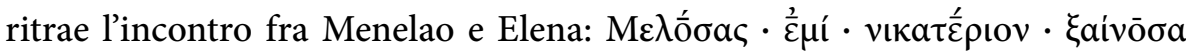

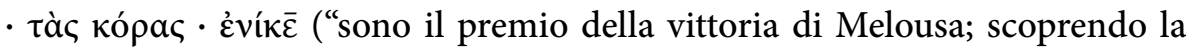
gamba vinceva le fanciulle"). ${ }^{41}$ Un ulteriore e assai interessante riferimento, più esplicito, a pratiche di consumo sociale del vino, cronologicamente riferibile alla metà circa del IV secolo a.C., viene da un'iscrizione dipinta sull'orlo esterno di un kantharos rinvenuto in frammenti nell'area dell'agorà di Metaponto (il che lascia supporre che l'oggetto fosse adoperato all'interno di un contesto pubblico, forse un banchetto; vid. fig. 7). ${ }^{42}$ Del testo di quest'epigrafe, non interamente conservato e consistente in un distico elegiaco, resta parte di

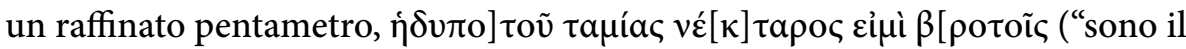

(1967, 226-227), $\mu[\grave{\varepsilon}] \nu$, l'unica delle altre che non credo possa essere esclusa sulla base della suddetta misurazione e che ovviamente va letta in correlazione al $\delta \dot{\varepsilon}$ posto al rigo successivo, resta quella che meglio di tutte si accorda con l'idea di un rimando sofisticato all'epica omerica.

40 Su questo punto vid. Murray 1994; 2009; 2011; Wecowski 2014, 127-139; 2017.

41 SEG XL, 900. L'interpretazione dell'epigrafe dipende, fondamentalmente, dalla traduzione del verbo $\xi a i v \omega$ (vid. LSJ s. v.). Considerando il significato comune di tale verbo, che fa parte del gergo tecnico della lavorazione della lana, indicando la cardatura, si è pensato che il testo faccia riferimento al suo supporto, indicandone la funzione di premio per una gara di tessitura di tipo rituale, collegata alla preparazione delle fanciulle al ruolo di sposa (Milne 1945). M. Guarducci (1967, 288-290), seguendo questa linea di pensiero, ha tradotto: "Sono il premio della vittoria di Melousa; cardando (la lana) vinceva le fanciulle”. Ė stato, però, convincentemente suggerito da M. Nafissi (1998, 36-37), in anni più recenti, un collegamento del documento tarantino con il simposio, le etere e la sfera ludico-erotica, vale a dire, in concreto, con una gara-esibizione erotica che implicasse il mostrare le gambe. Ciò ponendo in evidenza, da un lato, il fatto che la kylix, in quanto vaso a destinazione simposiale, difficilmente potesse essere idonea a costituire il premio per una fanciulla praticante "le ritirate virtù domestiche" (difficoltà già notata dagli editori precedenti), dall'altro, le significative e, nella tradizione, ben chiare valenze metaforiche di tipo sessuale possedute da $\xi \alpha i v \omega$, legate proprio allo scoprire le gambe, scostando le vesti, durante l'operazione di filatura. Tale tipo di xainein risulta sovente riferito, in maniera più o meno velata, alle etere ed al contesto del simposio, come indicano congiuntamente fonti di tipo letterario e iconografico. 
dispensatore ai mortali (?) del nettare dolce a bersi”), che, come rilevato da $\mathrm{M}$. Lombardo, trova suggestivi confronti sia letterari ${ }^{43}$ sia epigrafici. $^{44}$

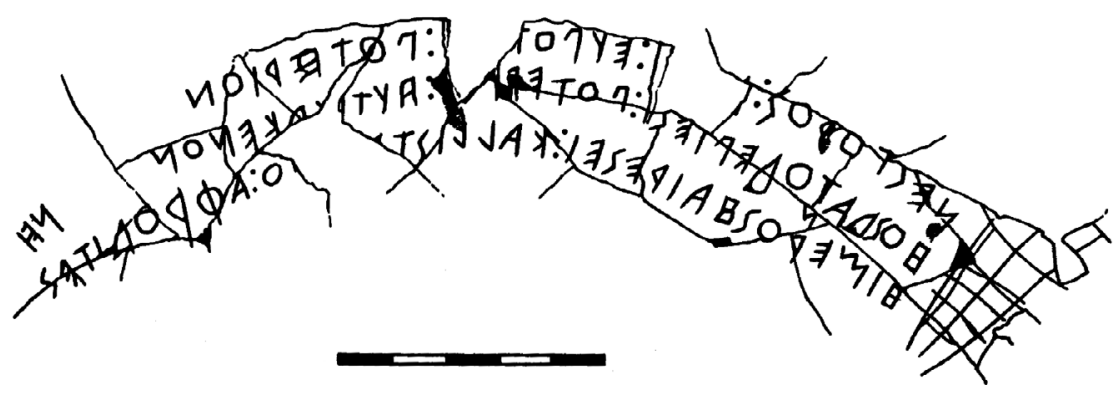

Fig. 5. Ischia, necropoli di S. Montano. Kotyle di fabbricazione rodia recante iscrizione metrica. Fac-simile dell'epigrafe. Ultimo quarto dell'VIII sec. a.C. (da Pavese 1996, fig. 1).

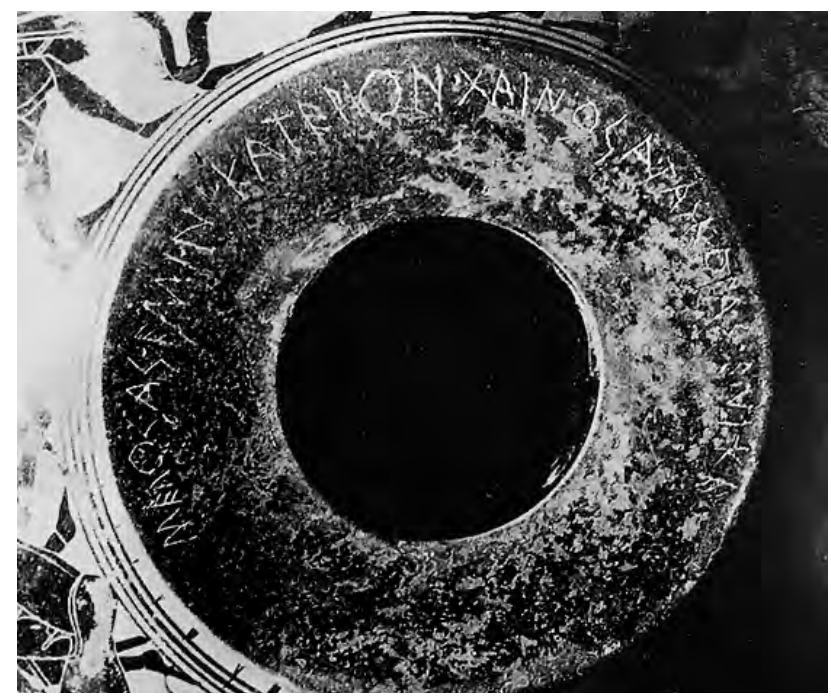

Fig. 6. Taranto. Kylix di Melusa. 540-520 a.C. Dettaglio dell'iscrizione graffita sotto al piede del vaso (da Guarducci 1967, 289, fig. 136).

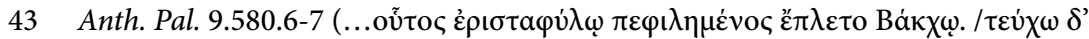

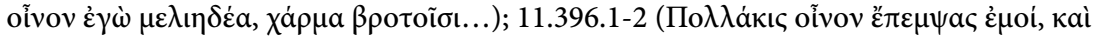

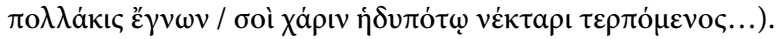

44 Ad es. negli ultimi due versi di un epigramma sepolcrale su stele marmorea, proveniente da un imprecisato luogo dell'Attica e databile fra il 410 e il 400 a.C. (Hansen 1983, 55, n.

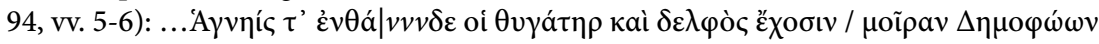

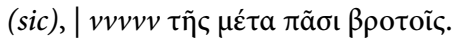




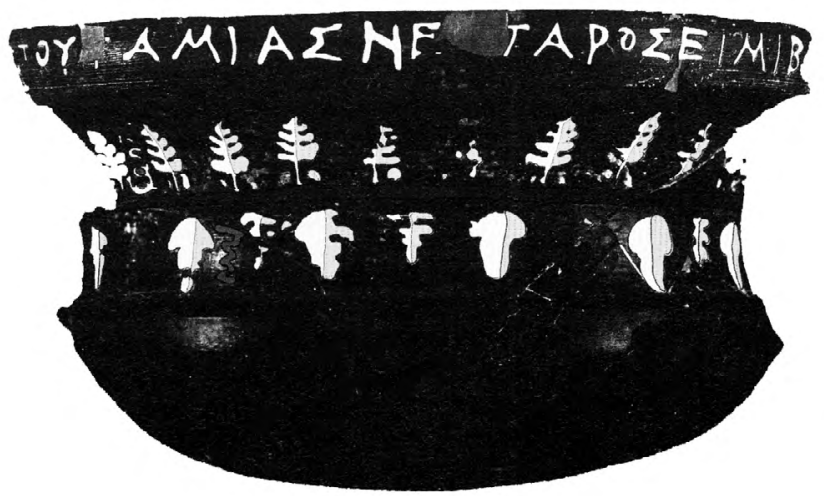

Fig. 7. Metaponto, area dell'agorà. Kantharos in frammenti recante un'iscrizione dipinta (da Lombardo e Frisone 2011, p. 291, fig. 4, con elaborazione grafica).

Il valore informativo e la rilevanza storico-epigrafica della coppa di $\mathrm{Ne}$ store sono legate non solo al tema del simposio ma anche a quello della nascita e della circolazione dell'epica omerica. Il collegamento che l'iscrizione pitecusana pone fra conoscenza dell'lliade e l'orizzonte culturale euboico figura fra gli elementi più importanti, che sono di ordine tanto epigrafico quanto letterario, sui quali si fonda l'ipotesi dell'esistenza di un coinvolgimento precoce dell'Eubea e dei suoi principali centri nella fase di immediata e più antica circolazione dei poemi omerici, dei quali alcuni tratti linguistici, che risultano innestati in una dizione già fissata e di differente ascendenza, sembrano riconoscibili appunto come euboici. ${ }^{45}$

Fra gli aspetti di rilievo più squisitamente epigrafici dell'epigrafe va, infine, sicuramente rimarcata, ai fini del nostro discorso, la raffinatezza dell'impaginazione $\mathrm{e}$, più in generale, dell'intera esecuzione, elementi già notati in passato. Rileviamo, in primo luogo, l'impressione di regolarità nella distanza fra le tre linee di scrittura e la loro corrispondenza ai versi (cosa, quest'ultima,

45 Cassio 1994, 1998. In tali lavori, lo studioso sostanzialmente discute la tesi di West (1988) secondo la quale il dialetto euboico avrebbe avuto un ruolo fondamentale, "normativo", nello sviluppo dell'epica omerica. Per alcuni, in particolare B.B. Powell (1991, 231-233. Vid. anche Robb 1994, 252-263), fautore dellorigine 'aedica' dell'alfabeto greco, il creatore di tale scrittura, un euboico, sarebbe anche l'autore della prima versione scritta dell'Iliade e dell'Odissea. Sulla possibile matrice euboica dell'Odissea vid. West 2014. Sulla genesi dell'epica omerica mi limito a rimandare, in una bibliografia comè noto vastissima e che ancora fa registrare la presenza di numerose ipotesi, anche molto distanti fra loro, in relazione al chi, al come, al quando e al dove, alla breve ma efficace messa a punto in West 2011. 
unica nella documentazione epigrafica arcaica riguardante testi in versi), ${ }^{46}$ poi la generale uniformità percepita nella dimensione, piccola (sono alte solo $0,5 \mathrm{~cm}$ ), delle lettere, nella loro spaziatura (anch'essa, in media, corrispondente a $0,5 \mathrm{~cm})^{47}$ e nel loro allineamento in verticale, non distante dalla precisione dello stile stoichedico, ${ }^{48}$ infine la presenza di segni di interpunzione a doppio punto. Siamo di fronte ad una competenza scrittoria di livello avanzato, ad una mano sicura, in grado di ben organizzare il testo nello spazio e che, per questo, sembra tradire, seguendo Immerwahr, la familiarità con supporti di tipo 'librario' e con la stesura di testi 'letterari' ${ }^{49}$

Spostiamo ora l'attenzione sul rapporto fra l'ambiente euboico campano e i contesti culturali italici, quello etrusco in particolare, ${ }^{50}$ richiamando due documenti significativi. Il primo è rappresentato dall'iscrizione pitecusana di Ame, su oinochoe di stile protocorinzio di imitazione locale, rinvenuta in una sepoltura femminile a inumazione e datata fra la fine dell'VIII secolo a.C. ed il primo decennio del secolo successivo: 'A $\mu \tilde{\bar{\varepsilon}} \varsigma \bar{\varepsilon} \mu$ í (fig. 8). ${ }^{51}$ Il nome del proprietario del vaso, piuttosto che derivare dall'ipocoristico di assai tarda attestazione ả $\mu \mu a ́$, che può significare "madre" o "nutrice" ed è noto anche come epiteto di Rhea e Demetra, ${ }^{52}$ può essere validamente collegato al gentilizio etrusco Amina, attestato a Pontecagnano nel tardo VI secolo a.C. ${ }^{53}$

\section{Jeffery 1961, 235-236.}

47 Valerio 2017, 12.

48 Jeffery 1961, 236.

49 Immerwahr 1990, 18-19. Su questo piano, la distanza fra l'iscrizione pitecusana e l'altro noto documento in versi di VIII secolo a.C. ad essa sovente accostato, l'iscrizione dell'oinochoe del Dipylon (SEG 3, 67; Guarducci 1967, 135-136; Catalin 2016, Binek 2017, Cardin 2017), pare davvero sensibile: tanto è ordinata e accurata la pianificazione grafica e l'esecuzione del testo euboico tanto risulta poco curata quella del testo ateniese, le cui lettere presentano elevate difformità nelle dimensioni, una spaziatura meno regolare $\mathrm{e}$ un non perfetto allineamento in orizzontale.

50 Su questo tema, oggetto di una bibliografia amplissima, mi limito a citare un acuto e originale studio di D. Ridgway (1997), centrato sulla diffusione in ambito etrusco di vasi e strumenti (la grattugia in bronzo in particolare) collegati alla preparazione e al consumo di bevande quali il vino e il kykeion, che rimandano tanto allorizzonte euboico quanto all'epica omerica. Vid. anche Bellelli 2012, con un ampio repertorio bibliografico.

51 Buchner, Bartonĕk 1995, 163-164; Arena 1994, 21-22; Colonna 1995, 329-331.

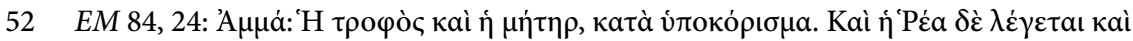

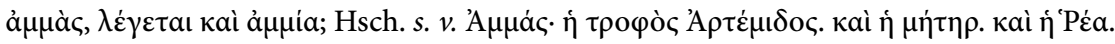
кaì ì $\Delta \eta \mu \eta \dot{\tau} \eta$. Tale ipotesi è sostenuta in particolare da R. Arena $(1994,22)$, che, infatti, trascrive $\mathrm{A}\langle\mu\rangle \mu \tilde{\varepsilon} \varsigma \bar{\varepsilon} \mu \mathrm{i}$.

53 CIE 8827. Vid. D’Onofrio 1984; Colonna 1995, 329-331; 2002. 


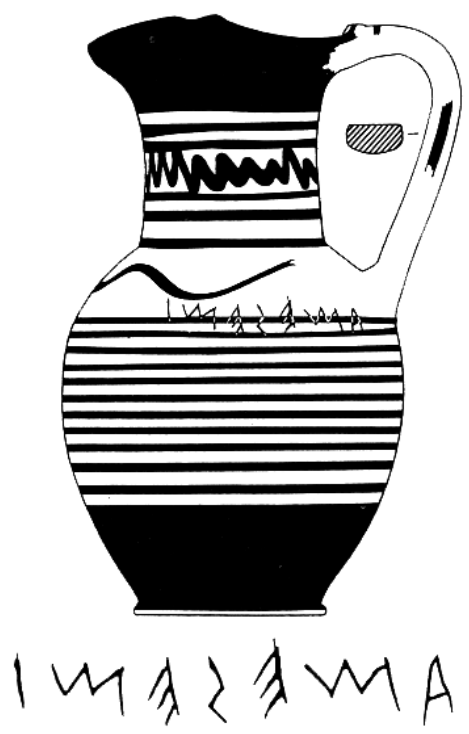

Fig. 8. Ischia, necropoli di S. Montano. Oinochoe protocorinzia d'imitazione locale, recante un'iscrizione di possesso graffita sulla parete. Fine dell'VIII secolo a.C. (da Buchner e Bartoněk 1995, 163, fig. 20).

Laltro documento di cui sopra è la nota e affascinante iscrizione cumana di Tataie, (fig. 9) su lekythos del protocorinzio medio risalente al secondo quarto del VII secolo a.C., ${ }^{54}$ avanziamo un poco, dunque, sul piano crono-

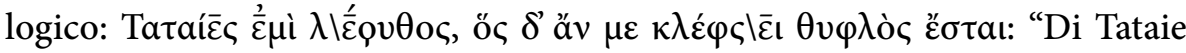
sono la lekythos. Chi mi rubi possa diventare cieco". Anche in questo testo "approssimativamente esametrico" 55 possiamo apprezzare un antroponimo femminile di origine non greca, Tataie, per il quale è stata suggerita, in maniera documentata, una matrice etrusca. ${ }^{56}$ Lepigrafe è preziosa, anche perché introduce una differente e affascinante categoria di testi, quelli legati alla sfera apotropaica, alla profilassi ed alla maledizione, di cui essa rappresenta l'esempio di più antico in ambito greco, diretto, nello specifico, alla difesa dal furto. ${ }^{57}$ Non è sfuggita, a chi si è cimentato con questo documento, la ricorrenza della formula ôc $\delta$ 'ö $v$, già vista nell'iscrizione della coppa di Nestore, che potrebbe essere presente anche nella sofisticata iscrizione in metri giambici di Hakesan-

54 SEG XL, n. 789; Buchner e Bartoňek n. C2.

55 Powell 1989, 340.

56 Colonna 1995, 331-332; 2002. Lo studioso fa leva sul confronto con l'iscrizione numes tataies mi, 'io (sono) di Nume Tataie' (Rix 1991, Cm. 2.48; Poetto e Facchetti 2009, 372).

57 Notevole risulta anche la più recente ma sempre arcaica iscrizione di Parmenone $\mathrm{e}$ Strimpone, graffita sul fondo di una coppa di produzione attica rinvenuta in una sepoltura di Pontecagnano, risalente al 500 a. C. circa (SEG 34.1019; 38.1012; 42.934; 53.1088;

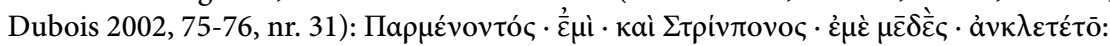
"Sono di Parmenone e Strimpone. Nessuno mi rubi". 
dros da Metone, su skyphos di produzione euboica databile fra la fine dell'VIII e gli inizi del VII secolo a.C., ${ }^{58}$ e che sicuramente compare, volta al femminile, su una kotyle cosiddetta a uccelli di produzione rodia, databile fra il 720 e il 710 a.C. e rinvenuta, purtroppo incompleta, a Eretria, ${ }^{59}$ qualificandosi, dunque, come caratteristica del repertorio epigrafico euboico più antico.
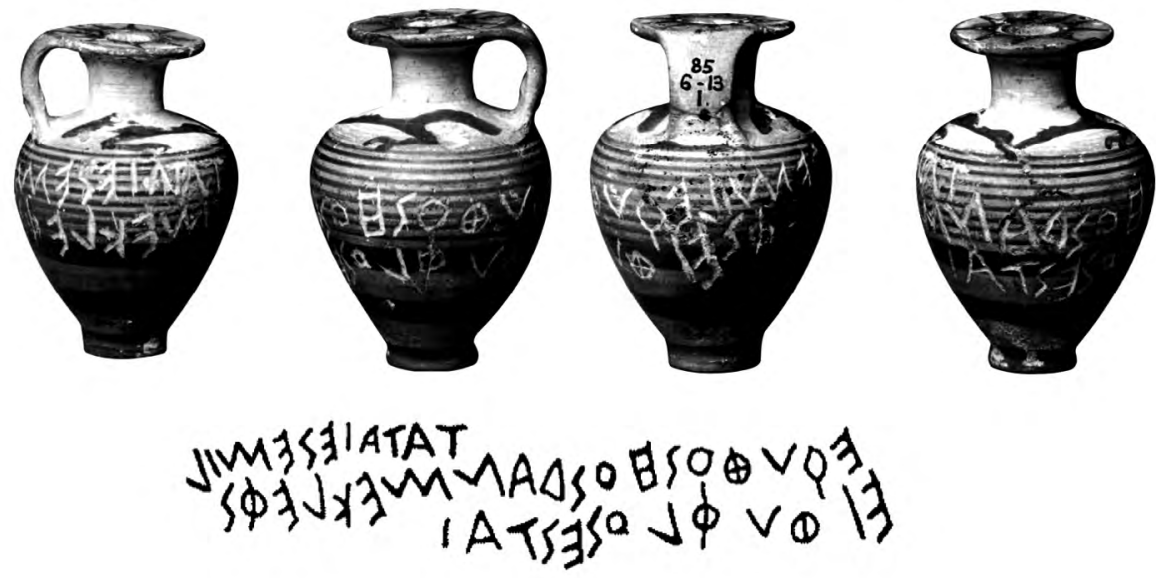

Fig. 9. Cuma. Lekythos protocorinzia, recante un'iscrizione graffita sulla parete. Secondo quarto del VII secolo a.C. (fac-simile da Buchner e Bartoněk 1995, 199, fig. C1; riprese fotografiche da Arena 1994, tavv. VIII-IX).

Ancor più emblematico, non solo sul piano della relazione fra il contesto greco e quelli italici ma anche in riferimento alla circolazione della scrittura ed alle complesse dinamiche ad essa collegate, risulta un altro eccezionale documento cumano. Mi riferisco alla lekythos d'imitazione locale del protocorinzio antico, risalente al primo quarto dell'VII sec. a.C. e rinvenuta in una sepoltura a inumazione, sul fondo della quale sono state tracciate, da mani differenti, due affascinanti e, in una certa misura, enigmatiche iscrizioni

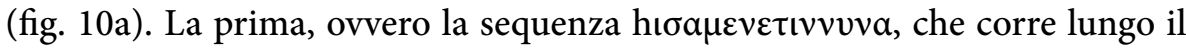
bordo con direzione retrograda, è stata letta sia in etrusco, da G. Colonna,

58 Bessios, Tzifopoulos e Kotsonas 2012, 339-343; Méndez Dosuna 2017, 246-247; Woo-

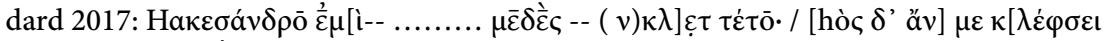
$\dot{\mathrm{o}} \mu(\mu)] \dot{\alpha} \tau \overline{0} v \sigma \tau \varepsilon \rho \bar{\varepsilon} \sigma[\varepsilon \tau] \alpha \underline{\mathrm{l}}$.

59 SEG 39.939; Johnston e Andriomenou 1989; Pavese 1996, 13-14: oө̣uнка[---] / vacat

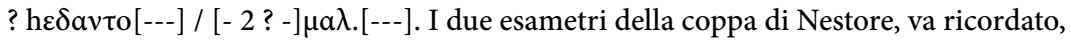
forniscono il più antico esempio epigraficamente noto di proposizione relativa ( $\mathrm{vid}$. Probert 2015, 202). 
sia in greco, da A. C. Cassio, ${ }^{60}$ il che trova una similitudine con quanto già visto a proposito dell'iscrizione dell'Osa. La prima delle due letture, che sembra quella più ampiamente condivisa, Hisa mene Tinnuna, da tradurre "Hisa Tinnuna fa (come dono)", individua un'iscrizione di dono il cui autore porta un nome etrusco, Hisa Tinnuna. Una tale formula bimembre, prenome più gentilizio, risulterebbe la più antica finora attestata in etrusco e sarebbe indizio di un rango elevato del personaggio, nel quale potrebbe essere visto un 'ospite d'onore' nell'ambiente cumano. ${ }^{61}$ La seconda lettura, invece, hĩ $\sigma \alpha \mu \varepsilon ́ v \bar{\varepsilon}$

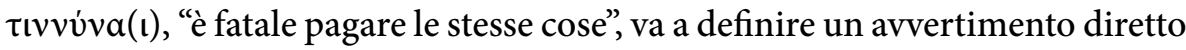
non tanto a scongiurare il furto dell'oggetto, considerato il suo non elevato valore, quanto la possibile violazione della sepoltura nella quale era posto. In tale forma il testo conterrebbe un'affermazione solenne del principio di "uguale retribuzione", ben noto nelle fonti letterarie, nella tragedia soprattutto, ed equivarrebbe, per contenuto, ad una maledizione. ${ }^{62} \mathrm{Nel}$ complesso, le tre iscrizioni considerate rappresentano un indizio piuttosto forte della circolazione come della presenza stanziale di individui di origine etrusca nell'ambiente euboico campano fra VIII e VII secolo a.C. ${ }^{63}$

Sulla citata lekythos cumana, oltre alla presunta iscrizione etrusca, vi è una seconda, curiosa epigrafe, verosimilmente più antica, costituita da due serie alfabetiche parziali (entrambe si interrompono allo zeta), eseguite con direzione ortograda e separate da una linea: $\mathrm{B} \Gamma \Delta \mathrm{FhZ} / \mathrm{AB} Г \Delta \mathrm{EFhZ} .^{64}$

60 Colonna 1995, 332-342; Cassio 1991-1993, 187-207. Cf. Buchner e Bartonĕk 1995, 201203. Già Ribezzo aveva proposto una lettura in senso greco poco fortunata: hí $\alpha$ $\mu \varepsilon ́ v \varepsilon$ $\tau ı v v \tilde{v} v a$, 'aspettati lo stesso ora' con riferimento alla violazione della sepoltura arcaica a inumazione del cui corredo il vasetto faceva parte (Ribezzo 1920, 75-76).

61 Colonna 1995, 340. Vid. anche Woudhuizen 1988-89.

62 Cassio 1995, 192, 194-198. La destinazione funebre del vaso è suggerita dalla presenza, sulla spalla, dell'immagine, dal carattere ctonio, del serpente. $\mu \dot{\varepsilon} v \bar{\varepsilon}$, nella lettura di Cassio, equivale a $\mu \varepsilon \dot{v \varepsilon \iota, \text { e } \tau ı v v v ́ v a ı ~ e ̀ ~ l ' i n f i n i t o ~ d e l l a ~ f o r m a ~ a t e m a t i c a ~ \tau i ́ v v \mu a ı, ~ p r o p r i a ~}$

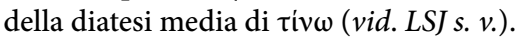

63 Per un approccio analitico al tema vid. il citato Woudhuizen 1988-89.

64 Vid. Buchner e Bartoněk 1995, 201-204 e l’accurata analisi in Ghinatti 2004-2005, 39-41. 


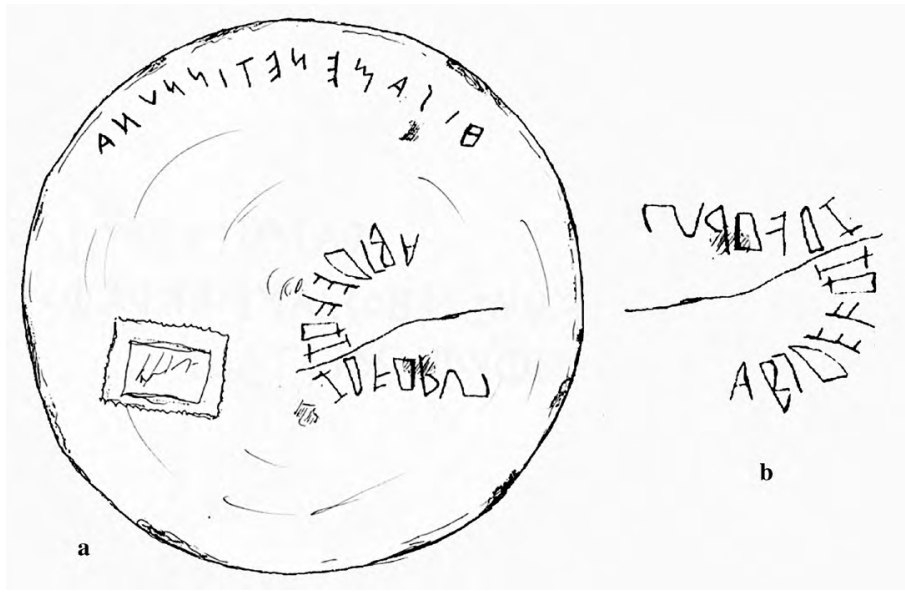

Fig. 10 a-b. Cuma. Lekythos protocorinzia d'imitazione locale, recante due iscrizioni graffite sul fondo. Primo quarto del VII secolo a.C. (fac-simile dell'autore).

Difficile risulta dire se le due sequenze siano state seguite dalla stessa mano ${ }^{65}$ e rendere conto del significato generale dell'insieme. La sequenza al di sotto della linea divisoria (fig. 10b), che potrebbe essere euboica, ${ }^{66}$ contiene tuttavia il segno di aspirazione nella forma chiusa e priva del trattino mediano, peraltro anteposto allo zeta, che evoca l'ambiente nassio in particolare (sia quello metropolitano sia quello coloniale), ${ }^{67}$ quella in alto, priva di alpha ed epsilon e contenente un segno identico per l'aspirazione, comincia con il beta a meandro di ascendenza corinzia. Che si tratti di un 'esercizio' in qualche modo riconducibile all'apprendimento della scrittura, oppure di un divertissement, che potrebbero essere stati realizzati tanto da un cumano quanto da un residente di origine corinzia ${ }^{68}$ nell'atto di riprodurre e confrontare la sequenza alfabetica euboica e quella corinzia, oppure ancora, secondo una suggestiva ma indimostrabile ipotesi di F. Ghinatti, di una sorta di codice di autenticazione del vaso, in cui la sequenza euboica indica la fabbricazione locale mentre quella corinzia il fatto che si tratti di un'imitazione di modelli

65 Secondo Jeffery 1961, 116-117 la sequenza posta in basso è scritta meglio rispetto a quella superiore.

66 Jeffery 1961, 116-117; Ghinatti 2004-2005, 41.

67 Il segno è attestato anche a Camarina, Cipro, Cnido, Egina (Ghinatti 2004-2005, 40-41).

68 Ormai da tempo è stata suggerita la presenza stanziale di elementi di origine corinzia nell'ambiente euboico campano, collegata alle attività delle officine ceramiche, in particolare quelle pitecusane (Neeft 1987, 59-65 e 309; Ridgway 1992, 62; Dell'Oro 2017, 172-175). 
allogeni, il documento suggerisce la circolazione, la compresenza e la conoscenza a Cuma di differenti modelli alfabetici greci (o almeno di alcune delle loro lettere caratteristiche).

Il documento, tuttavia, che più di ogni altro illumina ed esemplifica il rapporto che intercorre fra il mondo euboico e quello etrusco in relazione alla scrittura è l'alfabetario tracciato sulla riproduzione miniaturistica in avorio, di fattura etrusca, vetuloniese o vulcente, ${ }^{69}$ di una tavoletta scrittoria $^{70}$ (fig. 11), rinvenuta, unitamente ad alcuni manici di stilo e raschiatoi a spatola (che insieme alla tavoletta costituiscono un corredo da scrittura) e ad altri oggetti preziosi eburnei e aurei, all'interno di un lebete in bronzo pertinente alla sepoltura principesca nota come "Circolo degli avori", a Marsiliana d'Albegna. ${ }^{71}$ Loggetto è collocabile nel secondo quarto del VII secolo a.C. ${ }^{72}$ Questa la sequenza delle lettere, tracciate con direzione retrograda: $\alpha \beta \gamma \delta \varepsilon F \zeta h \theta \iota \kappa \lambda \mu v \boxplus o \pi M Q \rho \sigma \tau \cup X \Phi Y$. Si tratta di alfabetario fondamentalmente euboico, come indicano il delta rotondeggiante, il lambda calcidese e, soprattutto, il segno a finestra, variante del samek fenicio, che, oltre ad essere presente in quasi tutti gli alfabetari etruschi noti, ${ }^{73}$ è attestato ad Eretria all'interno di una sequenza alfabetica, purtroppo incompleta, ]田o $[$ [ , tracciata su un vaso monocromo risalente a un momento non precisabile dell'VIII sec. a.C. (fig. 12). ${ }^{74}$ Due lettere, tuttavia, sembrerebbero rimandare a tradizioni alfabetiche non euboiche, vale a dire a quella beotica, per quanto riguarda lo heta chiuso recante due tratti interni paralleli, ${ }^{75} \mathrm{e}$ a quella corinzia, in ragione del

69 Vid. Benzi 1966 e Martelli per la prima ipotesi, Cristofani 1971 per la seconda.

70 Normalmente questi strumenti, ben attestati in ambito vicino orientale (Wiseman 1955), erano fatti di legno, come quelle fortunosamente conservatesi nel fortilizio romano di Vindolanda, databili fra il I e il II sec. d.C. (Bowman e Thomas 1993; 1994).

71 La sepoltura conteneva tre copri inumati che, oltre che dal lebete e dal suo contenuto, erano accompagnati da armi in bronzo e un carro. Vid. Minto 1921.

72 Jeffery 1961, 236-238; Guarducci 1967, 228-229; Ghinatti 2004-2005, 44-48.

73 Jeffery 1961, 236-237; Pandolfini e Prosdocimi 1990; Wachter 2005.

74 Kenzelmann Pfyffer, Theurillat e Verdan 2005, 60, nr. 3. Il contesto di rinvenimento dell'oggetto, purtroppo disturbato, non consente una datazione più precisa.

75 Su tale lettera vid. Guarducci 1967 p. 144. Non è inverosimile ipotizzare che la presenza delle tre lettere citate possa essere collegata a quella, ormai accertata, di elementi di origine beotica, in particolare oropiana, alla quale sarebbe da ricondurre l'origine della designazione latina Graeci (Mele 2008, 102-103; Mazarakis-Ainian 2007, 28; Calce 2011, 126) e corinzia (Buchner 1981; Neeft 1987, 59-65, n. 176 e 177. Cf. Ridgway 1984, 85 e 163; 2012, 264-265, in cui si suggerisce l'esistenza di una situazione essenzialmente "demaratea" a Pitecusa "ben prima degli avvenimenti storici del 657") negli ambienti euboici campani. Già M. Guarducci $(1967,218)$ aveva messo in relazione il particolare 
gamma a uncino con angolo ottuso ${ }^{76}$ e del san. ${ }^{77}$ Per G. Colonna l'alfabetario di Marsiliana è il documento "più completo e fedele" di un alfabeto definibile come paleoetrusco, che sarebbe copia "di un alfabeto "cumano" del livello della coppa di Nestore" ${ }^{78}$ Il tema epigrafico e culturale di maggior rilievo che il documento pone, date le sue caratteristiche e considerato il luogo di rinvenimento, è quello del rapporto fra l'alfabeto greco, nella fattispecie quello euboico verosimilmente veicolato dall'ambiente coloniale campano, e la nascita di quello etrusco. ${ }^{79} \mathrm{M}$. Lejeune ${ }^{80}$ e A. Prosdocimi ${ }^{81}$ hanno chiaramente indicato come la sequenza di Marsiliana rappresenti, iconicamente, la radice di tale rapporto e il processo di trasmissione della scrittura dall'ambito culturale greco a quello etrusco, in quanto essa annovera e fornisce grafemi con i quali era possibile scrivere sia il greco sia l'etrusco, ovviamente all'opportuno e meditato variare della corrispondenza convenzionale fra tali segni e i fonemi delle due lingue. Sia che lo si consideri dal punto di vista greco, sia che lo si guardi, per così dire, con occhio etrusco, l'alfabetario di Marsiliana contiene, si tratta di un punto notevole, segni non usati nella concreta prassi scrittoria, vale a dire lettere cosiddette morte. In tal senso possiamo individuare, sul fronte greco, il segno a finestra e il citato san. Il primo è chiaramente una variante del samek fenicio la cui particolare forma è, forse, dovuta all'inglobamento nel disegno della lettera di tratti originariamente pertinenti a linee guida per

heta dell'alfabetario di Marsiliana con la presenza di elementi beotici a Cuma.

76 Colonna 1970; Dubois 1995, 16-17. Sull'alfabeto corinzio vid. Guarducci 1967, 170-171. L'uso di questo tipo di gamma risulta molto raro in ambito etrusco ed è attestato nelle iscrizioni dei noti kyathoi di produzione ceretana (vid. più avanti, n. 97) e nella serie alfabetica parziale (alfa adagiato, beta, gamma a uncino e delta tondeggiante, scritte con direzione retrograda ma orientate in senso ortogrado) incisa su un'anforetta a spirali del secondo quarto del VII secolo a.C. da Veio (vid. Colonna 2003; 2005), dove di norma viene impiegata la versione semilunata, attestata già fra la fine dell'VIII e gli inizi del VII secolo a.C. sulla cosiddetta kotyle Jucker da Tarquinia (Jucker 1969).

77 Colonna 1970, 667-668.

78 Colonna 1970, 663.

79 Bellelli e Benelli 2018, 28-36; Benelli in questo volume. Sia consentito rimandare, su questo tema anche a Boffa 2016.

80 Per Lejeune 1983, 12, "Eubéens et Étrusques usent, au moment de la transmission de l'écriture des uns aux autres, d'un abécédaire commun: même répertoire de signes, ranges dans le même ordre".

81 Pandolfini e Prosdocimi 1990, 19-21 e 195-205 
la scrittura, ${ }^{82}$ il secondo deriva verosimilmente dal sempre fenicio tsade ${ }^{83} \mathrm{Il}$ segno a finestra costituisce una lettera morta anche sul fronte etrusco, insieme ai segni che, in greco, sono beta, delta, omicron.$^{84}$ Ancorché morte, ognuna di tali lettere, la cui sopravvivenza negli alfabetari etruschi va ricondotta alla volontà di rendere tali sequenze "interoperabili", ovvero adatte alla scrittura di più lingue ${ }^{85}$ era comunque pronunciata nella recita della sequenza e, dunque, era comunque associata ad un nome e ad un valore fonetico, prova ne sia, il fatto che nel passaggio dell'alfabeto da Etruschi a Latini, i citati segni beta, delta, omicron, non adoperati dai primi, vennero invece usati dai secondi con valori pressoché identici a quelli posseduti in ambiente greco. ${ }^{86}$
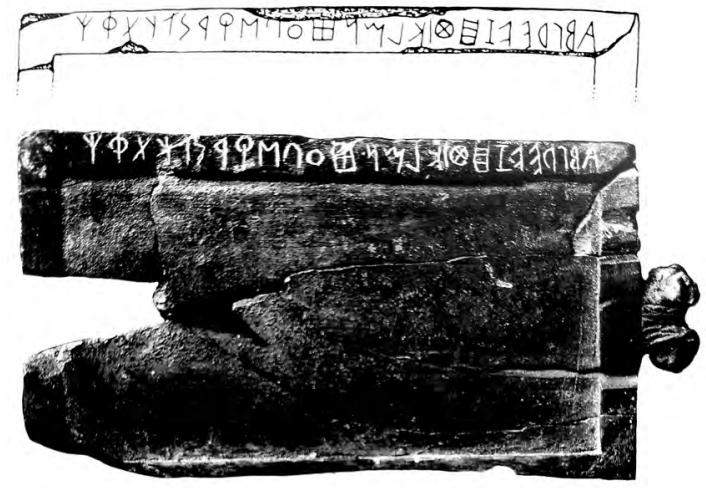

Fig. 11. sinistra: Marsiliana d'Albegna (Manciano, GR), dalla sepoltura principesca nota come "Circolo degli avori". Tavoletta scrittoria eburnea miniaturistica, recante un alfabetario. Secondo quarto del VII secolo a.C. (fac-simile e ripresa fotografica rispettivamente da Pandolfini e Prosdocimi 1990, 20 e tav. I).

Fig. 12. destra: Eretria, frammento di vaso monocromo recante elementi di una sequenza alfabetica. VIII sec. a.C. (da Verdan, Kenzelman Pfyffer e Theurillat 2012, 181, n. 5).

82 Pandolfini e Prosdocimi 1990, 199 e 206. Poco convincente, invece, è l'ipotesi di F. Ghinatti, secondo cui l'alfabetario di Marsiliana era strutturato in maniera tale da poter servire come sequenza modello per due sistemi alfabetici: quello rosso euboico e quello blu orientale (Ghinatti 2004-2005, 47). La mancanza dell'omega, infatti, come lo stesso Ghinatti riconosce, che allepoca della stesura del documento era già in uso (Guarducci 1967, 101), costituisce un ostacolo difficilmente aggirabile.

83 Su questo punto importante e controverso dell'origine dell'alfabeto vid. l'accurata disamina in Slings 1998.

84 Rix, 1984, 213; Benelli 2006, 21-24.

85 Su questo punto vedi il contributo di Enrico Benelli in questo volume.

86 Né è possibile pensare, com'è stato ormai chiarito (Wallace 2011,11-12) ad una diretta influenza dell'alfabeto greco su quello latino. Su questi punti è fondamentale Pandolfini e Prosdocimi 1990, 231. Il segno a finestra, seguendo questa linea di ragionamento, doveva verosimilmente conservare il valore originario fenicio. 


\section{Circolazione e diffusione della scrittura: canali, soggetti, percorsi dell'alfabetizzazione, competenze scrittorie}

La rassegna di documenti presentata, pur nella sua brevità, è sufficiente a porre in risalto alcuni degli elementi e dei problemi principali legati alla nascita e al primo sviluppo della cultura epigrafica nel contesto magnogreco e, più in generale, greco. Il primo, come detto, risiede nel fatto che tali fenomeni siano in realtà tutt'uno con quello più ampio della nascita dell'alfabeto e della cultura epigrafica greca, il che ne accresce enormemente il respiro e l'importanza, ponendo allo stesso tempo il problema dei "canali di circolazione" che all'interno dell'intero orizzonte greco veicolarono la scrittura e i fenomeni grafici e funzionali ad essa collegati. Con grande forza emerge il ruolo dei centri euboici, delle loro attività e delle loro fondazioni. Vale la pena ricordare a tal proposito che oltre all'iscrizione dell'Osa conosciamo solamente altre due iscrizioni greche (considerando le sole sequenze di lettere in cui possiamo riconoscere un testo) risalenti alla prima metà dell'VIII secolo a.C., rinvenute nei centri euboici metropolitani di Lefkandi ( [---] $\sigma \alpha \leftarrow$ oppure $\rightarrow A \mu[---]$, su frammento di vaso geometrico, vid. fig. 13) ed Eretria (] $\theta$ oı [, su frammento di anfora, vid. fig. 14) ${ }^{87}$ Possiamo essere sicuri del fatto che, ce lo indica, in particolare, il doppio alfabetario cumano (ma anche quello di Marsiliana, se ha ragione il citato Dubois sulla presenza di lettere di impronta corinzia), differenti modelli alfabetici greci fossero contemporaneamente noti e circolanti, in ambiente euboico e verosimilmente altrove nel mondo greco (e non solo), ${ }^{88}$ e che di essi si potesse di volta in volta fare sintesi e selezione. D'altro canto, $\mathrm{i}$ due documenti (che al pari di tutti gli alfabetari possiamo definire metascrittorii, ovvero riconducibili a una riflessione teorica sulla scrittura), indicano anche come l'insieme delle conoscenze sulla scrittura fosse, nel caso specifico dei rispettivi estensori, più ampio di quanto richiesto per poter concretamente scrivere nella propria lingua. Tale situazione trova una sua fondamentale definizione nel concetto di "corpus dottrinale" sviluppato da A. Prosdocimi,

87 Sul documento lefkandiota e quello eretriese vid. rispettivamente Popham, Sackett e Themelis 1980, 90, nr. 102; Kenzelman Pfyffer, Theurillat e Verdan 2005, 75, nr. 64. Si escludono dal computo le ricorrenze di singole lettere o segni para-alfabetici. 
indicativo del bagaglio complessivo delle conoscenze relative ad un sistema di scrittura, comprendente segni grafici e regole per il loro uso (corrispondenze ai fonemi e indicazioni ortografiche). ${ }^{89}$

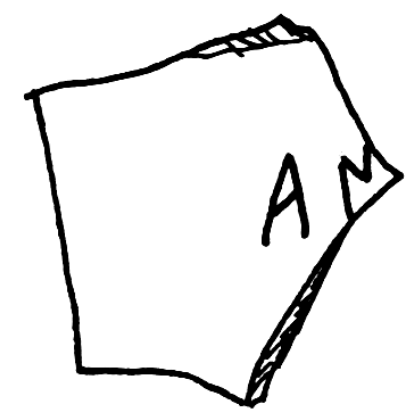

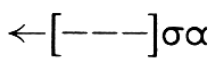

or

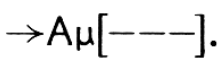

Fig. 13 (sinistra). Lefkandi. Frammento di vaso geometrico recante un'iscrizione incompleta. Primo quarto dell'VIII secolo a.C. ca. (da Powell 1991, 124, n. 3).

Fig. 14. Eretria. Frammento d'anfora recante un'iscrizione incompleta. Prima metà dell'VIII secolo a.C. (da Verdan, Kenzelman Pfyffer e Theurillat 2012,181, n. 3

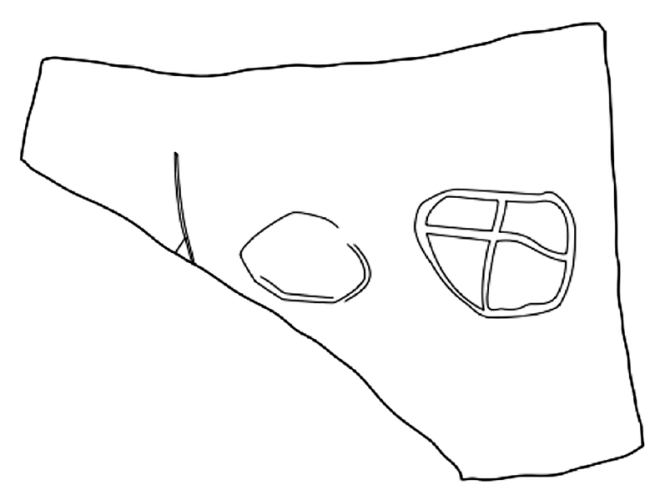

Mettere a fuoco i canali di circolazione della scrittura significa anche individuare i concreti utenti della scrittura, considerati nella loro dimensione sociale. Un primo elemento da porre in rilievo consiste nel fatto che, nel panorama della documentazione epigrafica greca più antica, quella pertinente all'VIII secolo a.C., sia essa di ambiente metropolitano o coloniale,

89 Pandolfini e Prosdocimi 1990, 188-189. Il perpetuarsi della presenza di lettere (con relativi nomi e suoni) estranee alla selezione di segni concretamente adoperata per scrivere è indicativa di ciò che Prosdocimi ha definito la "prospettiva dei maestri", ovvero del fatto che, nelle prime fasi di vita dell'alfabeto, comprendenti, sia nel caso greco sia in quello etrusco, una fase di trasmissione/adattamento e una di prima diffusione, esso venisse guardato dalla prospettiva della scrittura modello (Pandolfini e Prosdocimi 1990, 164-165). Per una revisione critica del concetto di corpus dottrinale vid. il già citato contributo di Enrico Benelli nel presente volume, che ne conferma la sostanziale validità precisandone, allo stesso tempo, limiti di opportunità di utilizzo. 
non troviamo documenti che consentano di collegare in maniera forte l'uso della scrittura alle differenti élites, diversamente da quanto abbiamo potuto osservare in ambito etrusco, con riferimento al documento di Marsiliana in particolare. ${ }^{90}$ La scrittura greca, per come ci appare dai documenti che possediamo, sembra appannaggio dei livelli inferiori della stratificazione sociale dei centri coinvolti, anche nel caso in cui si abbia a che fare con testi complessi e di ascendenza epica e "letteraria" come quello della coppa di Nestore. Siamo anche in grado di riconoscere, inoltre, una specifica categoria professionale collegata all'uso della scrittura alfabetica già nella fase iniziale della sua storia: quella degli artigiani. Le competenze scrittorie di questi ultimi emergono attraverso quella particolare specie documentaria rappresentata dalle firme, di cui abbiamo visto l'esempio più antico (la cui provenienza da Pitecusa, alla luce di quanto osservato, difficilmente può essere considerata casuale). Gli artigiani possono aver senzaltro rappresentato un veicolo di trasporto e distribuzione della scrittura, stante la loro riconosciuta, elevata e costante mobilità all'interno dell'intero contesto Mediterraneo. ${ }^{91}$

Da segnalare, per complessità e articolazione formale, l'iscrizione del ceramista Nikomachos, tracciata, anteriormente alla cottura, su un piccolo cippo tetragono di terracotta rinvenuto nel territorio di S. Mauro Forte, attuale comune della provincia di Matera situato nell'entroterra dell'antica fondazione achea di Metaponto (fig. 15). Tale territorio, vicino al corso della Salandrella, naturale via di comunicazione verso la costa dell'attuale Basilicata, distante

90 Un'indicazione analoga retituiscono i già citati kyathoi (vid. n. 76) in bucchero di origine ceretana, rinvenuti soprattutto in ambito settentrionale a partire dalla metà del VII secolo a.C. (Bagnasco Gianni 2008; Maggiani 2009; Maras 2010). Questi manufatti, che costituiscono oggetti di prestigio funzionali alla pratica del dono in ambito aristocratico (Sciacca 2004), recano iscrizioni apposte dagli stessi artigiani, che, dunque, erano parte integrante del prodotto e rispondevano ad una precisa richiesta da parte del committente. Sul tema vid. anche Maras 2012a.

91 Su questo punto vid. D’Agostino 2003, secondo cui il rapporto fra scrittura e decorazione vascolare sarebbe maturato proprio in ambiente euboico e la firma d'artista costituirebbe una vera e propria esibizione dellorgoglio professionale e della competenza scrittoria. Alla presenza di artigiani di origine vicino-orientale in ambiente greco, lo ricordiamo, è stata collegata la trasmissione della scrittura da Fenici a Greci e la genesi dell'alfabeto (Burkert 1992, 9-40). La mobilità degli artigiani greci, segnatamente euboici, e la loro presenza negli ambienti italici rappresenta uno degli elementi chiave nel contatto e nell'interazione fra i rispettivi ambiti culturali (D’Agostino 1985; Della Fina 2004; Bonaudo 2008-2009; Bellelli 2010; Benelli 2004; 2014). Esemplare e strettamente attinente alla situazione delineata è l'arrivo dei tre fictores greci, Eucheir, Eugrammos e Diopos a Tarquinia, intorno alla metà del VI secolo a.C. (vid. n. 20). Vid. Ridgway $2009 \mathrm{e}$ la recente e ampia disamina in Ampolo 2018. 
circa cinquanta chilometri, è poco noto sul piano archeologico. Si possono, allo stato attuale delle conoscenze, apprezzare tracce sparse di popolamento di età arcaica, che non consentono di individuare un centro abitato ma solo resti di fattorie e piccole necropoli, pertinenti alla cultura autoctona. ${ }^{22}$ L'oggetto, risalente alla fine del VI sec. a.C., è alto $40 \mathrm{~cm}$ e presenta un incavo rettangolare sotto la base, il che lascia supporre che fosse fissato a un supporto ${ }^{93}$ Esso mostra evidenti affinità tipologiche con i cippi in pietra squadrati provenienti dal santuario di Apollo a Metaponto, recanti dediche alla divinità. ${ }^{94}$ Il testo, in alfabeto acheo, si dipana su cinque linee di scrittura collocate singolarmente su tre lati e in coppia su uno, e va così letto, seguendo la soluzione offerta da

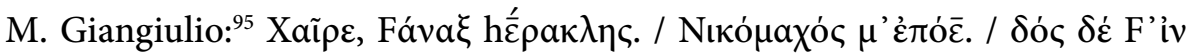

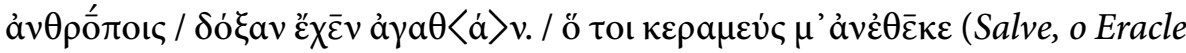
Signore. / Nicomaco mi fece. / Concedi che lui fra gli uomini / abbia buona fortuna. / Il vasaio mi ha dedicato). Siamo di fronte ad una sequenza di "cola organizzata nei tipici ritmi kat'enoplíon della più antica lirica greca", ${ }^{96}$ che formano un sofisticato e articolato insieme di elementi comprendente: un'invocazione ad Eracle, che trova un puntuale confronto nell'inno archilocheo a tale divinità; ${ }^{97}$ la firma dell'artista Nicomaco; una richiesta di buona reputazione, che riprende alla lettera un emistichio dell'elegia alle Muse di Solone $;{ }^{98}$ e una dedica fatta dallo stesso ceramista. Il tutto è collocato all'interno della cornice comunicativa dell'iscrizione parlante.

92 Tramonti 1983; Frisone 2010.

93 IG XIV 652; SEG 52, 2002, 958.

94 Su questi ultimi vid. Antonetti, De Vido e Drago 2013.

95 Giangiulio 1993, 34-35 (con ampia e acuta discussione del documento e ricca bibliografia), che a sua volta si basa su Duhoux 1984.

96 Giangiulio 1993, 34. Più precisamente, si tratterebbe, nell'ordine, di tre hemiepes maschili (ll. 1, 2, 3), un hemiepes femminile (1. 2) e un enoplio (1. 4). Per una differente (e, a mio avviso, meno convincente) lettura dei caratteri metrici del testo, che, basata su una differente ricostruzione della successione delle linee di scrittura, individua un distico i cui elementi sono separati da un hemiepes, vid. Arena 1989, 30-31.

97 Archiloc. Fr. 324 West. Vid. Hansen 1983, 215, nr. 396; 1985; Arena 1989, 31.

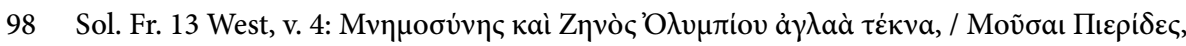

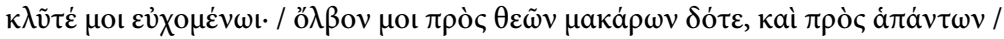

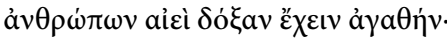



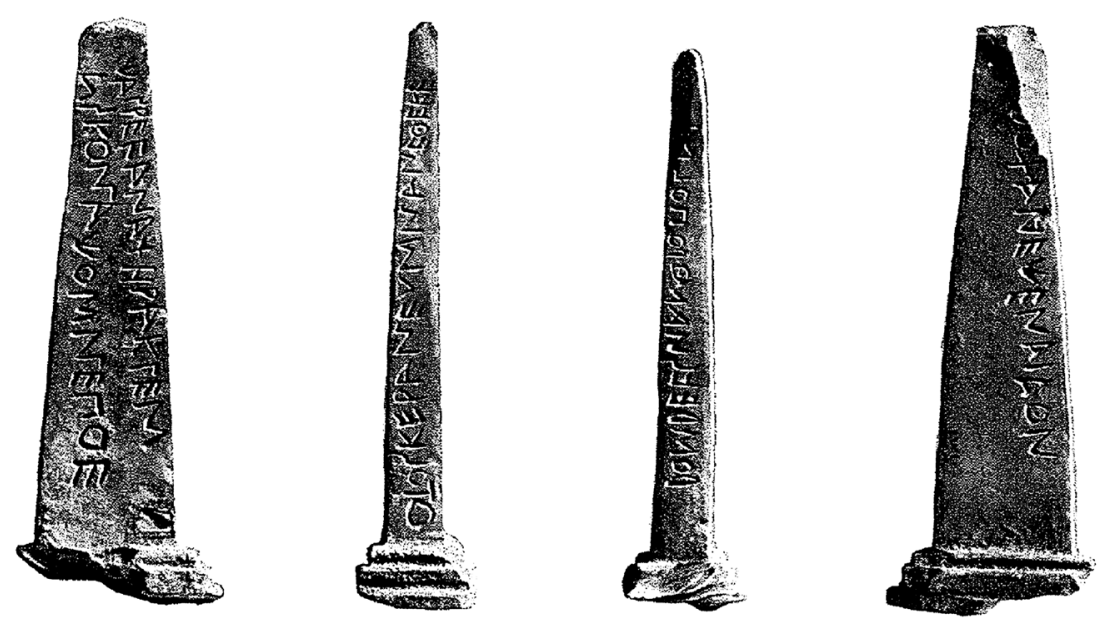

Fig. 15. S. Mauro Forte. Piccolo cippo tetragono in terracotta, recante la

firma dell'artigiano. Fine del VI sec. a.C. (da Guarducci 1974, 556, fig. 232).

Nell'estensore, seguendo la convincente ricostruzione proposta del citato Giangiulio, va visto non un 'semplice vasaio' ma un artigiano dallelevata capacità, la cui attività principale era verosimilmente legata non tanto alla produzione della ceramica ma a quella della coroplastica, di tenore tecnico e artistico più elevato, e che intratteneva, in forza di tale capacità, rapporti con committenze locali di elevato livello sociale. ${ }^{99} \mathrm{Nicomaco}$, che, come lascia intendere il luogo di rinvenimento del documento, operava in un contesto di frontiera rappresentando egli stesso un elemento mediatore fra lorizzonte culturale greco e quello locale, esibisce un profilo culturale elevato e sofisticato, testimoniato dai riferimenti colti del testo, e in una certa misura sorprendente sia rispetto alla professione esercitata ${ }^{100}$ sia rispetto all'ambiente nel quale l'oggetto venne prodotto. ${ }^{101} \mathrm{Il}$ nostro ha evidentemente pianificato l'esecuzione

99 Giangiulio 1993, 42-43.

100 E ancora materia di discussione la valutazione della consistenza metrica delle epigrafi di età arcaica. La polarizzazione delle opinioni in proposito, vede, da un lato, chi tende a riconoscere in maniera ampia e articolata (Gentili 1967, 39; Di Tillio 1969; Moranti 1972; Gentili e Giannini, 1977, 19 e 22) o addirittura amplissima (Gallavotti 1979) una forma metrica e, in non pochi casi, una dignità stilistica (con agganci soprattutto alla lirica) nelle iscrizioni arcaiche, dall'altro chi invece tende alla soluzione opposta (in particolare Hansen 1983; 1984).

101 Giangiulio 1993, 41. Poiché Eracle non rientra fra gli elementi mitico-religiosi collegati alla sfera dell'artigianato, va ipotizzato che il suo culto avesse una particolare e differente funzione all'interno del contesto locale (Giangiulio 1993, 44-47. Cf. Giacometti 2005, 131-137). Non è banale ricordare, seguendo quanto recentemente illustrato da R. Bianco 
dell'iscrizione e accuratamente calcolato il rapporto fra lo spazio disponibile e la lunghezza prevista dello scritto. In funzione di tale calcolo, e per evitare che lo spazio venisse a mancare, egli ha iniziato a scrivere su una delle due facce più larghe, ivi tracciando due linee in direzione progressiva, per poi proseguire ruotando loggetto in senso antiorario (guardandolo in piedi) e seguendo l'andamento bustrofedico, vale a dire cambiando la direzione della scrittura al cambiare del lato. Vanno notate alcune discrasie nellesecuzione delle lettere, che in alcuni casi mostrano un orientamento opposto a quello congruente con la direzione della scrittura (le prime due lettere, $n i$ e iota, della seconda

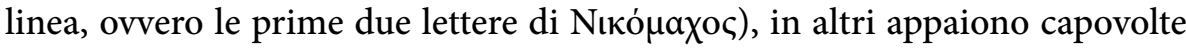
( $\delta \dot{\varepsilon}$ F' $i v$ all'interno della terza linea; la quarta linea inizia con la sequenza ö to $\kappa \varepsilon$ - correttamente eseguita mentre le restanti lettere sono sottosopra).

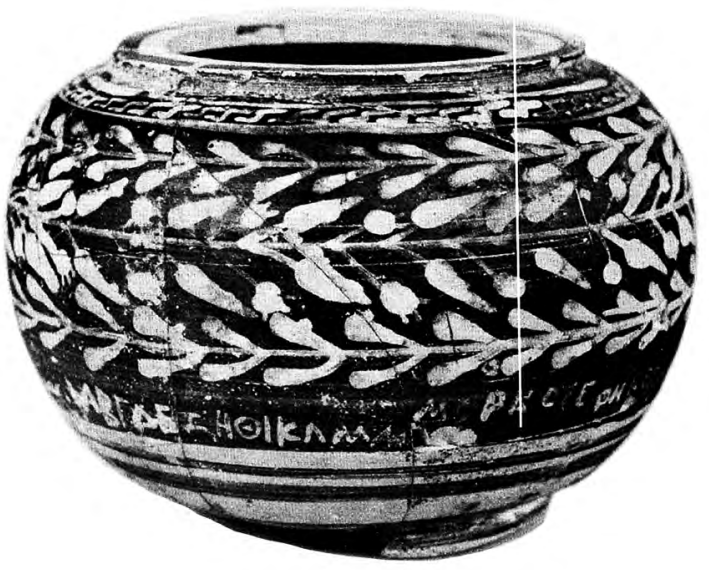

Fig. 16. Gravina, località Botromagno. Pisside di produzione locale recante un'iscrizione dipinta. Fine del V - inizi del IV secolo a.C. (da Santoro 1978, tav. IX a).

Il documento, però, che più di ogni altro rappresenta il legame fra la scrittura, il suo insegnamento e gli artigiani ceramisti è l'iscrizione di Morkos, dall'insediamento peuceta in località Botromagno di Gravina (fig. 16). ${ }^{102} \mathrm{Si}$ tratta di unepigrafe dipinta su una pisside di produzione locale prima della cottura della stessa e, dunque, redatta dall'artigiano. L'oggetto, di cui si ignora l'esatto contesto di rinvenimento, è databile, sulla base di considerazioni tipologiche, fra la fine del V e gli inizi del IV secolo a.C. ${ }^{103}$ Questo ne è il testo:

(2018), che gli artigiani della ceramica sono fra i principali protagonisti della diffusione del mito Eracle fra le popolazioni italiche in contatto con gli insediamenti magnogreci.

102 SEG 54, 955, n. 3. Su questo documento rimando a Boffa 2017.

103 C. Santoro $(1978,226)$, primo editore dell'iscrizione, riferisce di un rinvenimento "in 


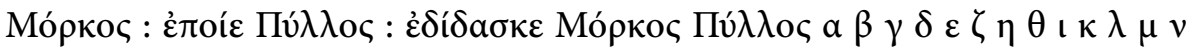

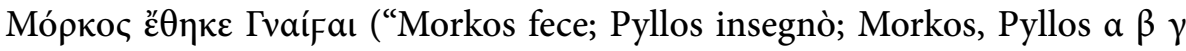
$\delta \varepsilon \zeta \eta \theta \iota \kappa \lambda \mu \nu ;$ Morkos donò a Gnaiva"). A prescindere dalle sue possibili traduzioni e dalle relative e diverse possibilità di attribuzione dei ruoli ai due personaggi maschili, Morkos e Pyllos, i cui nomi hanno ascendenze illiriche ${ }^{104}$ il testo riunisce una firma (غ̇đoíc), un riferimento diretto all'insegnamento $\left(\delta\llcorner\delta a ́ \sigma \kappa \omega)\right.$, un alfabetario parziale e una iscrizione di dono $\left({ }^{\prime} \theta \eta \kappa \varepsilon\right)$ rivolta a un personaggio femminile il cui nome ha origine osche. ${ }^{105}$ Seguendo l'ipotesi interpretativa di A. Small, in Morkos va visto l'artefice del vaso e l'autore dell'iscrizione, in Pyllos il suo maestro sia per l'arte ceramica sia per la scrittura, ritenendo che l'alfabetario, sebbene parziale, non possa che far allusione proprio all'insegnamento di quest'ultima. ${ }^{106}$ Lo stesso Morkos dona l'oggetto alla donna. ${ }^{107}$ Il documento gravinese, dunque, ci presenta due artigiani di origini illiriche che lavorano in un centro peuceta, si esprimono anche per iscritto in greco e hanno familiarità con una donna di origini osche. Tutto ciò, oltre a ben esemplificare il ruolo di crocevia detenuto dall'insediamento di Botromagno (nel quale va vista l'antica $\Sigma \mathrm{\iota} \delta$ íov, poi $\Sigma \iota \lambda \beta$ íov, ${ }^{108}$ che sorgeva in un'area in stretto contatto a sud con i centri greci di Taranto e Metaponto, a ovest

una tomba dell'agro di Gravina in circostanze non precise".

104 Santoro 1978, 240-268. Cfr. Fraser e Matthews 1997, ss. vv. Morkos; Pyllos).

105 Si tratta del femminile di Гvaĩoc, che nel caso specifico presenta il digamma intervocalico, secondo un uso ben documentato, per il periodo che ci interessa, sia in ambiente tarantino sia in quello messapico. Su questi elementi vedi Santoro 1978, 268-273. Vid. anche Fraser e Matthews 1997, s. v. Tvaĩfa. Per le ricorrenze del nome nell'epigrafia osca vid. Rix 2002, 139.

106 Small 2004, 32. Contra Santoro 1978, 29, secondo il quale l'alfabetario ha una funzione magica e l'iscrizione non allude all'insegnamento della scrittura

107 La traduzione riportata è sostanzialmente quella proposta da C. Santoro (1978), che risulta la più ampiamente condivisa. Ho tuttavia preferito rendere $\varepsilon$ $\theta \eta \kappa \varepsilon$ con "donò" piuttosto che con "dedicò". Dal momento, infatti, che possiamo escludere che Gnaiva sia il nome di una divinità, l'iscrizione configura un dono e non una dedica, come invece ritenuto da Santoro. Tí $\theta \eta \mu$, del resto, ha un significato più ampio e generico rispetto ad

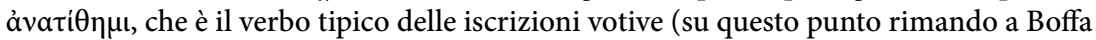
2017). S. Marchesini (2013, 28), ha invece suggerito: "Morkos; fece Pyllos; insegnò Morkos; Pyllos $\alpha \beta \gamma \delta \varepsilon \zeta \eta \theta \iota \kappa \lambda \mu$ v; Morkos dedicò a Gnaiva”. Si tratta di una traduzione che ha il pregio di rispettare la posizione delle interpunzioni e che ribalta i ruoli dei due personaggi maschili, Morkos e Pyllos. La mia preferenza va, tuttavia, alla soluzione offerta da Santoro (che riteneva errato il posizionamento delle suddette interpunzioni, fenomeno non certo infrequente nell'epigrafia greca), perché trovo più logico pensare che colui che ha donato il vaso a Gnaiva debba essere stato anche l'artefice dell'oggetto e dell'iscrizione.

108 Diod. Sic. 20.80, 1. Vid. Giannotta 1989; Mangieri 1995. 
con i centri lucani parlanti osco e a est con quelli peuceti costieri, a loro volta legati ai centri costieri illirici ${ }^{109}$ ) sottolinea con forza, da un lato, il rapporto degli artigiani ceramisti con l'uso e l'insegnamento della scrittura, suggerendo come la bottega del vasaio potesse anche essere luogo di alfabetizzazione e formazione scrittoria, ${ }^{110}$ dall'altro la centralità della scrittura nei processi di scambio e interazione culturale fra il mondo greco e quello italico. ${ }^{111}$

Le iscrizioni di Tataie e di Melousa, in precedenza illustrate, alle quali

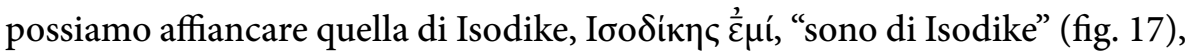
dipinta su un peso da telaio di forma piramidale proveniente da Siri, risalente alla prima metà del VI secolo a.C., ${ }^{112}$ ci portano, d'altro canto, a considerare il rapporto fra il mondo femminile e la scrittura, sul quale in tempi recenti si è insistito con maggiore frequenza. Com'è stato ben puntualizzato, tale rapporto non è di facile definizione, a causa di limiti quantitativi e qualitativi presenti nella documentazione disponibile, sia essa letteraria, epigrafica o iconografica. ${ }^{113} \mathrm{~A}$ tal proposito, per quanto riguarda la seconda categoria, si registra costantemente la difficoltà di non poter essere certi dell'identità dell'estensore, che potrebbe anche non corrispondere ai personaggi femminili di riferimento. È il caso anche delle tre iscrizioni citate. I documenti di Isodike e di Melousa rimandano ad attività e situazioni che possiamo considerare tipiche del mondo femminile ma che sono rispettivamente collegate a contesti e ruoli sociali ben diversi: da un lato l'oikos e le attività muliebri proprie della donna da marito, la cui educazione è orientata alla $\sigma \omega \varphi \rho o \sigma u ́ v \eta$, dall'altro il simposio e la seduzione, elementi propri delle etere (aderendo all'ipotesi interpretativa di Nafissi). Diverso, invece, è il tenore dell'iscrizione di Tataie, il

109 Su questo punto vid. le considerazioni in Boffa 2017, 304-305.

110 Su questo tema Rebillard 1989. Va segnalato anche un altro documento di grande interesse rinvenuto ad Altamura, non lontano da Botromagno, dunque, e grosso modo coevo. Si tratta di una coppetta a vernice nera che reca una serie di sei lettere separate da interpunzioni, forse una indicazione di bottega, tracciate ad argilla ancora fresca dal ceramista, e un alfabetario di matrice tarantina, redatto dopo la cottura da una mano diversa e accompagnato da altre quattro lettere. Vid. Boffa 2017, 299-304.

111 Per una panoramica sul rapporto fra l'alfabeto greco e le lingue italiche in area magnogreca vid. Poccetti 2015.

112 Guarducci 1974, 350-351. L'iscrizione è redatta in alfabeto ionico milesio. Notevole la presenza del segno heta chiuso.

113 Dillon 2013; Wolicki 2015, 310-311 (quest'ultimo fa particolare riferimento alla documentazione iconografica ateniese di età classica). Appare, più precisamente, difficile valutare in termini quantitativi il coinvolgimento delle donne nell'uso della scrittura, che, in ogni caso appare decisamente marginale rispetto a quello degli uomini, soprattutto nell'ambito dell'età arcaica. 
cui più rilevante risvolto storico e culturale risiede nell'essere testimonianza della presenza stanziale di donne di origini italiche nell'ambiente cumano, ${ }^{114}$ con tutto ciò che questo può comportare in termini di interazione culturale $\mathrm{e}$ di costruzione dell'identità dei singoli e della comunità. ${ }^{115}$

Fig. 17. Antica Siris. Peso fittile da telaio di forma piramidale, recante, dipinta, l'indicazione del possesso. Prima metà del VI secolo a.C. (da Guarducci 1974, 351, fig. 121).

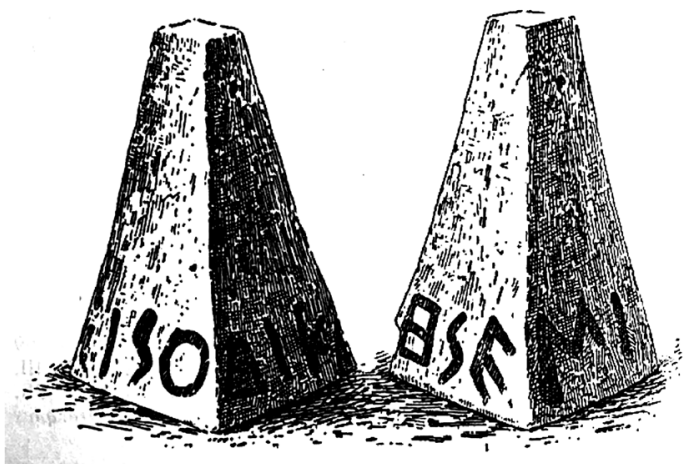

Fra le difficoltà più grandi che si incontrano nello studio della nascita e della diffusione dell'alfabeto all'interno della articolata e variegata realtà culturale e sociale del mondo greco arcaico vi sono quelle legate, da un lato, alla valutazione dell'estensione quantitativa dell'uso della scrittura, sia nella dimensione diacronica sia in quella sincronica, dall'altro alla valutazione dell'estensione qualitativa della stessa, intendendo con ciò i livelli di competenza scrittoria degli scriventi. La prima è condizionata da limitazioni non aggirabili che riguardano la documentazione e, in particolar modo, ciò che non abbiamo e che, per definizione, non potremo mai avere: gli scritti su supporti deperibili. Su questi ultimi si è ragionato in numerose occasioni nel tentativo di definirne gli ipotetici contorni, fino a farne il perno di una delle teorie della nascita dell'alfabeto ( $\mathrm{mi}$ riferisco all'ipotesi di Heubeck sulla origine commerciale di tale scrittura). ${ }^{116}$ In ogni caso, la logica suggerisce che, partendo da un punto zero, collocabile nel momento della sua creazione, l'alfabeto abbia via via conquistato nuovi utenti, seguendo la progressione ben chiarita dalla teoria della diffusione delle innovazioni sviluppata da E. Rogers,

114 Su questo tema vid. Coldstream 1993; Hall 2004; Ziskowski 2007; Saltini Semerari 2016.

115 Si tratta di un tema molto dibattuto in tempi recenti, soprattutto in riferimento agli insediamenti coloniali greci. Vid., a proposito di Pitecusa e Cuma, Mermati 2012; 2014; Donnellan 2016a; 2016b.

116 Heubeck 1979. Secondo lo studioso i documenti epigrafici più antichi rappresentano solo una piccola frazione della reale produzione scritta, che doveva perlopiù essere collegata a supporti deperibili come pelli, papiri, tavolette lignee, e al mondo del commercio. Su questi temi $c f$. Lombardo 1987. 
di cui qui provo a utilizzare principi e strumenti con intento descrittivo. ${ }^{117}$ Ad nucleo di innovators, nei quali vanno individuati i responsabili dell'adattamento al greco della scrittura fenicia e, dunque, i primi utenti del nuovo sistema, ${ }^{118}$ avrà fatto seguito un più ampio gruppo di early adopters, seguito da una early e una late majority e da un gruppo di laggards. ${ }^{119}$ La decisione di adottare la scrittura, ovvero di adattare al greco il sistema di scrittura fenicio, sarà passata attraverso una fase di "consapevolezza dell'innovazione”, vale a dire di conoscenza passiva della scrittura orientale. Tale conoscenza avrà generato il desiderio della sua adozione solo nel momento in cui essa sarà stata percepita come rilevante in relazione alla situazione sociale e culturale degli innovators. Quindi, dopo una fase di "persuasione", all'interno della quale si sarà formata un'idea favorevole all'adozione, si sarà giunti alla fase di implementazione e di concreto uso. ${ }^{120}$ Il successo e la progressiva diffusione della nuova tecnologia ${ }^{121}$ all'interno del contesto greco antico sarà stato decretato dalla sua buona rispondenza a cinque fattori: "il vantaggio relativo", ovvero

117 Formalizzata nel 1962 da E. Rogers e nata all'interno degli studi di sociologia rurale (Rogers 1983, 38-86), di cui lo studioso era docente alla Ohio State University, la teoria mira spiegare, con un approccio che è allo stesso tempo antropologico e sociologico, come e perché nuove idee e tecnologie si propagano all'interno di un sistema sociale. I suoi elementi chiave, sebbene scaturiscano dall'analisi di contesti sociali e culturali moderni e da una documentazione sicuramente più ampia e variegata rispetto a quella disponibile per il mondo antico, possiedono una validità universale, dal momento che essi individuano meccanismi basilari del comportamento umano. Essi riguardano i fattori in gioco nella diffusione dell'innovazione (innovation, adopters, communication channel, time, social system), le fasi del suo divenire (knowledge, persuasion, decision, implementation, confirmation), il tipo di decisione che è alla sua base (optional, collective o authority innovation-decision) e le categorie socioculturali che concretamente la realizzano (innovators, early adopters, early majority, late majority, laggards). La diffusione delle innovazioni è allo stesso tempo un processo particolare di comunicazione, in cui il messaggio riguarda una nuova idea, e una forma di cambiamento sociale (Rogers 1983, 1-7). Per un riesame della teoria e un approfondimento riguardante la sua interazione con la social network analysis, vid. Greenhalgh et al. 2005; Von Pape 2009. Applicata e testata nel corso del tempo in innumerevoli settori della ricerca antropologica e sociale, la teoria non risulta ancora impiegata, a mia conoscenza, in relazione allo specifico problema dell'introduzione e della diffusione della scrittura.

118 Poco importa se a realizzare tale adattamento sia stata una singola persona o un ristretto gruppo. Entrambe le possibilità possono rientrare nell'ipotesi monogenetica della nascita dell'alfabeto greco, che vede la realizzazione di quest'ultimo in un unico evento e in un solo luogo. Meno probabile pare, oggi, l'ipotesi poligenetica (anche da me in passato caldeggiata). Vid. su questi punti le considerazioni in Janko 2017.

119 Rogers 1983, 240-251. La consistenza numerica, espressa in termini percentuali, delle suddette categorie è il 2,5\% per gli innovators e il 13,5\% per ognuna delle altre.

120 Rogers 1983, 164-175.

121 La scrittura è, a tutti gli effetti, una tecnologia (Ong 2002, 80-82). 
l'innovatività rispetto alla situazione precedente; la "compatibilità", con i valori e i bisogni di potenziali utenti; la "complessità" nell'apprendimento e nell'uso; la "sperimentabilità", ovvero la possibilità di provarne l'uso con limitate basi di apprendimento; 1" osservabilità", vale a dire il grado di visibilità dei risultati dell'innovazione all'interno del contesto sociale nella quale avviene l'uso. ${ }^{122}$

Questo quadro teorico ben si accorda con le conoscenze epigrafiche, e più in generale storiche, sociali e culturali, che possediamo in relazione al tema. I Greci non partirono da zero e non realizzarono un sistema scrittorio ex novo ma presero a modello e modificarono ${ }^{123}$ una scrittura di cui dovevano avere da tempo nozione, essendo almeno dal X sec. a. C. assidui frequentatori (in particolare gli Euboici) di numerosi luoghi del Vicino Oriente e accogliendo, a loro volta, presenze orientali anche di tipo stanziale. ${ }^{124}$ L'alfabeto, poi, ben risponde ai cinque sunnominati fattori: è relativamente facile da apprendere $^{125}$ e può essere sperimentato con immediatezza in svariate condizioni, per differenti e anche molto limitati usi ${ }^{126}$ e con l'impiego di un amplissimo

122 Su questi punti vid. Rogers 1983, 210-240.

123 Il che realizza, nella prospettiva sviluppata da Rogers, una reinvenzione (Rogers 1983, 175-182).

124 In una bibliografia amplissima e assai variegata mi limito a rimandare a Braun 1982; Fantalkin 2006; Van Dongen 2008. A Eretria e a Pitecusa, del resto, sono stati rinvenuti documenti epigrafici in scrittura semitica, formalmente affini ma di ben difficile interpretazione, l'uno su frammento ceramico di produzione locale databile fra la fine del IX e gli inizi dell'VIII secolo a.C., l'altro su anfora adibita a enchytrimos, riferibile al terzo quarto dell'VIII sec. a.C. I rispettivi testi recitano $k p l s ̌$ [, nel quale è stato visto il suggestivo ma non dimostrabile tentativo di scrivere con il sistema fenicio una parola greca,

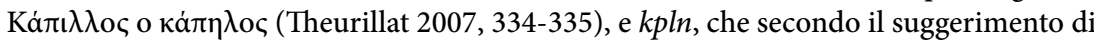
G. Garbini potrebbe significare, in aramaico, "il doppio" (Garbini 1978, 130-135). Da Pitecusa viene anche unaltra iscrizione aramaica, di lettura incerta, databile alla fine dell'VIII sec. a.C. (Garbini 1978, 140-142), così come da Eretria provengono anche gli eccezionali paraocchi bronzei per cavallo recanti una dedica in aramaico del sovrano di Damasco Hazaël, pertinenti all'ultimo quarto del IX sec. a.C. (Amadasi Guzzo 1987; Bron e Lemaire 1989). Sui documenti in scrittura semitica da contesti greci vid. il recente contributo di G. Bourogiannis 2015.

125 Lo sforzo principale nell'apprendimento dell'alfabeto è collegato non tanto alla memorizzazione dei segni e dei relativi valori fonetici, il cui numero è limitato, quanto alla composizione delle sillabe e dei gruppi consonantici. Vid. sul tema Johnson 2015; West 2015.

126 I differenti livelli di abilità nell'uso della scrittura e nella composizione del testo che si evincono dalla documentazione presa in esame, che spazia, per l'VIII secolo a.C., dalla semplice scrittura del proprio nome al genitivo per l'indicazione del possesso alla stesura di un'iscrizione sofisticata come quella coppa di Nestore, sono strettamente collegati sia alle esigenze comunicative del singolo individuo sia alle aspettative che in quest'ultimo ripone il contesto sociale. Non tutti, in definitiva, avevano bisogno di usare la scrittura 
repertorio di strumenti e superfici semplici e facilmente reperibili. Impiegato su oggetti non deperibili, ovvero nella sua dimensione epigrafica, esso è in grado di acquistare un elevato livello di visibilità, fattore che ha un'influenza determinante nello sviluppo di una diffusa percezione della sua presenza all'interno del contesto sociale in cui viene adoperato. ${ }^{127} \mathrm{La}$ novità stessa dello strumento, nonché la flessibilità e l'efficacia che esso possiede nell'assecondare un numero potenzialmente illimitato di esigenze comunicative, molte della quali sorgono proprio in conseguenza della sua adozione, presentano ricadute positive sia in termini di vantaggio relativo sia di compatibilità.

L'epigrafia greca presenta un salto molto deciso, in relazione al numero di documenti noti, fra la prima e la seconda metà dell'VIII sec. a.C. Nell'ambito della prima, infatti, possiamo inquadrare soltanto i tre già visti documenti di ascendenza euboica, mentre nel contesto della seconda le iscrizioni note sono almeno una sessantina, ${ }^{128}$ il che suggerisce un'esplosione dell'uso della scrittura in tale periodo. ${ }^{129}$ Inoltre, nel corso dell'età arcaica, fra VIII e VII sec. a.C., si coglie una generale evoluzione verso la complessità compositiva e tematica delle epigrafi, che, partendo dai semplici e brevi testi legati al possesso, alla dedica sacra e alla firma, porta ad una maggiore frequenza di testi più strutturati e all'emersione di esigenze espressive e comunicative via via nuove e più sofisticate, che possiamo apprezzare, per quel che riguarda l'ambito magnogreco, nel noto dischetto bronzeo da Cuma (fig. 18), risalente alla metà del VII secolo a.C., che attesta l'esistenza di un ora-

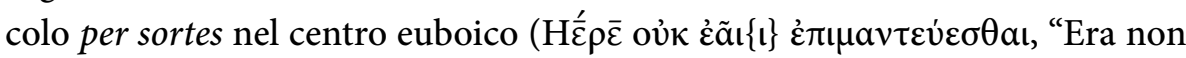
permette che si torni a consultare l'oracolo"), ${ }^{130}$ oppure nel piccolo aryballos globulare del corinzio medio (fig. 19) da Taranto, databile a poco dopo il 570 a.C., su cui è graffita una iscrizione funeraria che riecheggia l'epica omerica,

con lo stesso livello di complessità compositiva e di abilità esecutiva, cosa che accade anche nelle moderne società dell'alfabetizzazione di massa. Su questo punto e sul fondamentale concetto di alfabetizzazione funzionale applicato alla realtà greca antica sono particolarmente utili le acute riflessioni in Thomas 2009.

127 Il caso delle epigrafi su ceramica è, in tal senso, esemplare. Vid. su questo punto Osborne e Pappas 2007; Boffa c. s.

128 La recensione dell'evidenza edita non è cosa agevole. Orientativamente, possiamo calcolare che alle quaranta iscrizioni indicate da A. Bartoněk nel 1994 (Bartoněk 1994, 113) si debbano aggiungere le ventidue epigrafi rinvenute a Methone la cui cronologia oscilla fra la fine dell'VIII e gli inizi del VII sec. a.C. (Bessios, Tzifopoulos e Kotsonas 2012, 337-369) e alcuni documenti da Mende e Oropos recensiti dal SEG.

$129 C f$. Luraghi 2010, 72.

130 SEG 40 816; Guarducci 1967, 229-230. Vid. anche la rilettura di Kajava 2010. 
per il riferimento alla felicità della dimora eterna (Hom. Od. IV, 561-568), e sembra anticipare elementi propri della spiritualità orfico-pitagorica (Tò

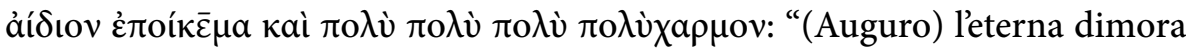
e molto, molto, molto gioiosa"), ${ }^{131}$ oppure, ancora, nella curiosa e decisamente più prosaica iscrizione in alfabeto acheo dipinta su una casseruola in ceramica, di cui non si conosce il luogo di rinvenimento e che risale al 500 a.C. circa

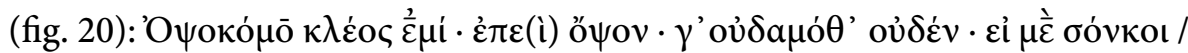

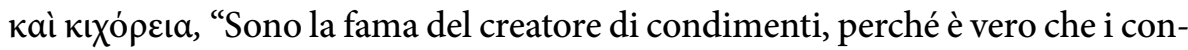
dimenti sono privi di valore a meno che non includano cicerbita e cicoria". ${ }^{132}$

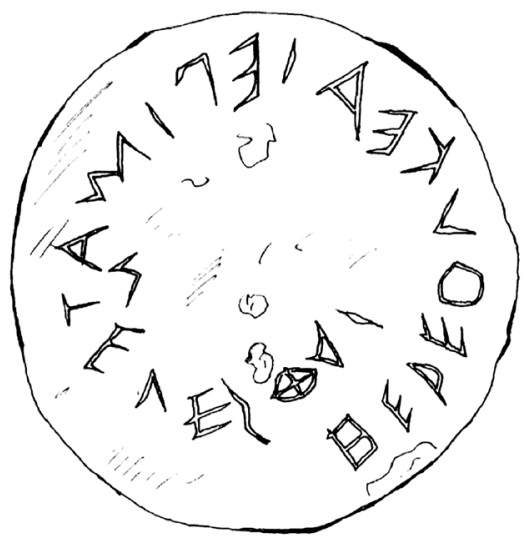

Fig. 18. Cuma. Dischetto bronzeo recante iscrizione incisa. Metà del VII secolo a.C. (Fac-simile dell'autore).

Fig. 19. Taranto. Aryballos globulare del corinzio medio, recante un'iscrizione. 570 a.C. ca. (da Ferrandini Troisi 2015, 114).
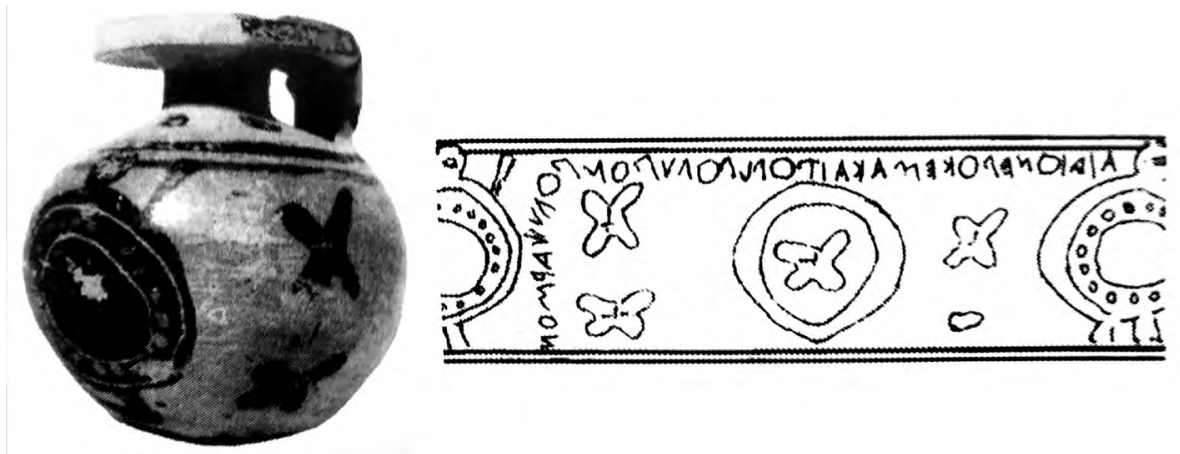

131 Ferrandini Troisi 2015, n. 129.

132 Dubois 2002, 178-180, nr. 103. L'estensore aveva iniziato a scrivere il testo, con direzione retrograda, nello spazio compreso fra le anse, tracciando le lettere fra due linee parallele

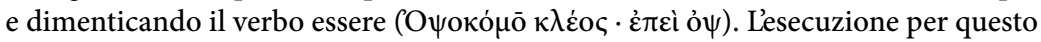
motivo fu interrotta e ripresa daccapo più in alto, immediatamente al di sotto dellorlo. Il documento è notevole sia per gli aspetti epigrafici (la presenza del segno dello psi a stella e dell'interpunzione a punto singolo), sia per quelli linguistici (il termine 
Fig. 20. Casseruola in ceramica, di provenienza ignota ma attribuita all'Italia meridionale, recante un'iscrizione dipinta in alfabeto acheo. 500 a.C. ca. (da Dubois 2002, 178, nr. 103).

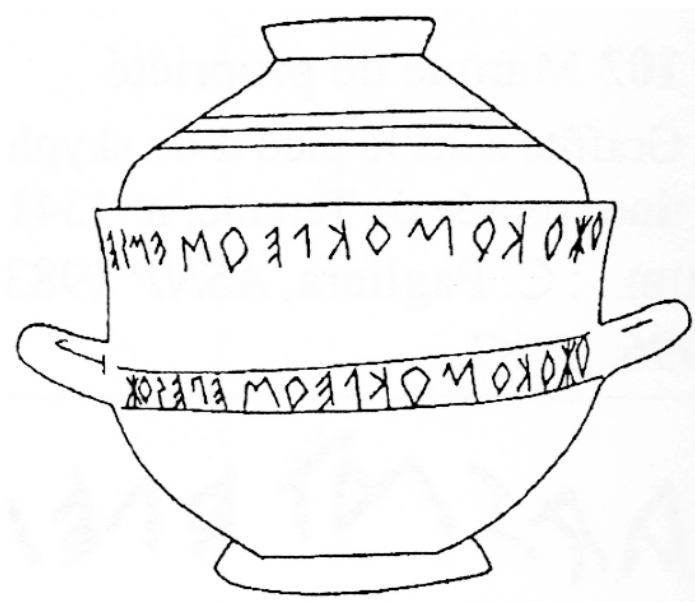

Tali esigenze, fra VII e VI secolo a.C. oltrepassano la sfera privata per andare a interessare anche quella pubblica, assecondando l'evoluzione politica e istituzionale che si registra all'interno del mondo greco. ${ }^{133}$ L'iscrizione su lastra di carparo da Torricella (fig. 21), dal territorio di Taranto, risalente alla metà del VI secolo a.C., che riporta un elenco di oggetti che si riferiscono verosimilmente ad una cerimonia religiosa ed al conseguente pasto rituale, forse

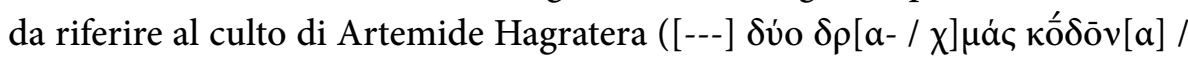

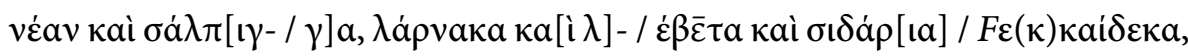

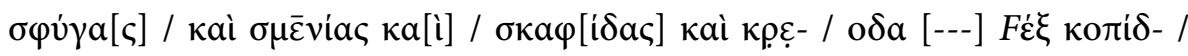

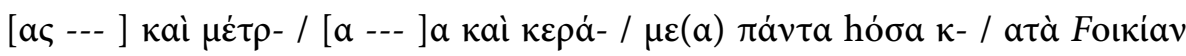

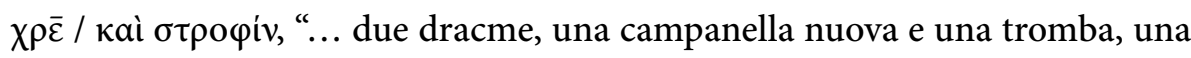
cassa e un lebete e sedici attrezzi di ferro, martelli e asce e recipienti..., sei coltelli e misure ... e tutti quanti gli oggetti di ceramica necessari per una casa e una benda") ${ }^{134}$ e il noto trattato fra i Sibariti e i loro alleati e i Serdaioi

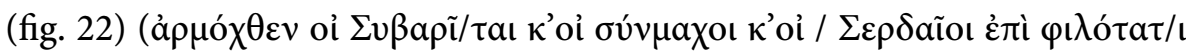

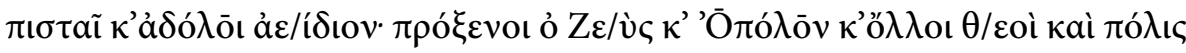

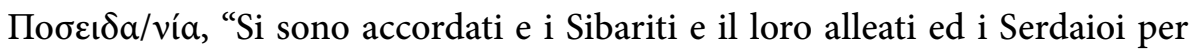

'О

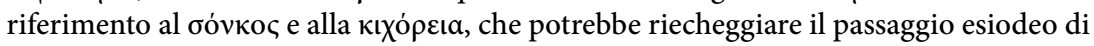
Op. 40-41), sia per quelli tematici e comunicativi, decisamente insoliti. Il vaso conteneva probabilmente una salsa a base di cicerbita e cicoria, bollite e messe a macerare nellolio d'oliva.

133 Val la pena ricordare che la più antica legge pervenutaci, che impone un limite all'iterazione della carica di kosmos, viene da Drero e risale alla seconda metà del VII sec. a.C. Vid. De Rossi 2017.

134 Ferrandini Troisi 2015, n. 123. 
stringere un patto di amicizia fedele e senza inganno, per sempre; testimoni: Zeus e Apollo e gli altri dei e la città di Poseidonia”), generalmente ascritto alla seconda metà del VI secolo a.C. e rinvenuto a Olimpia, rappresentano due elementi di notevole interesse storico ed epigrafico in tale ottica. ${ }^{135}$

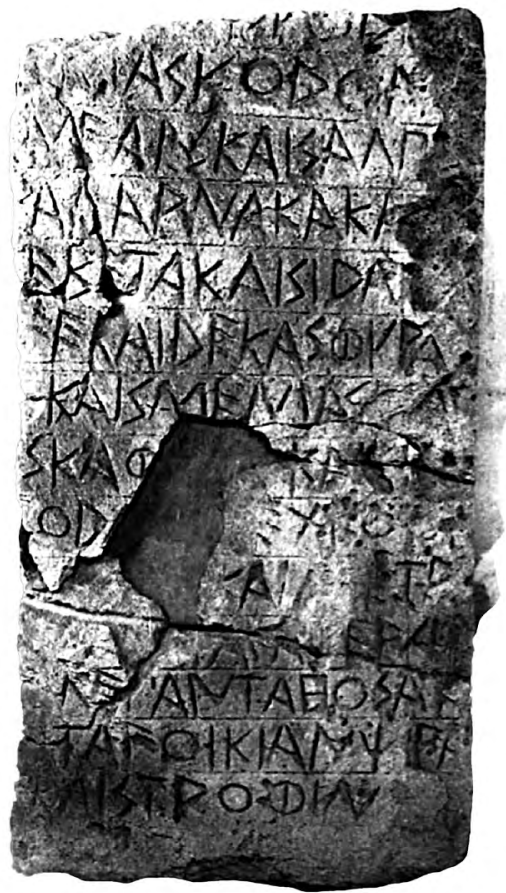

\author{
Fig. 21. Torricella, località "Pezza della \\ torre". Lastra di carparo con iscrizione. \\ Metà del VI secolo a.C.).
}

Fig. 22. Olimpia. Tabella bronzea recante il testo di un trattato fra i Sibariti e i loro alleati e i Serdaioi. Seconda metà del VI secolo a.C. (da Guarducci 1969, 541, fig. 169).

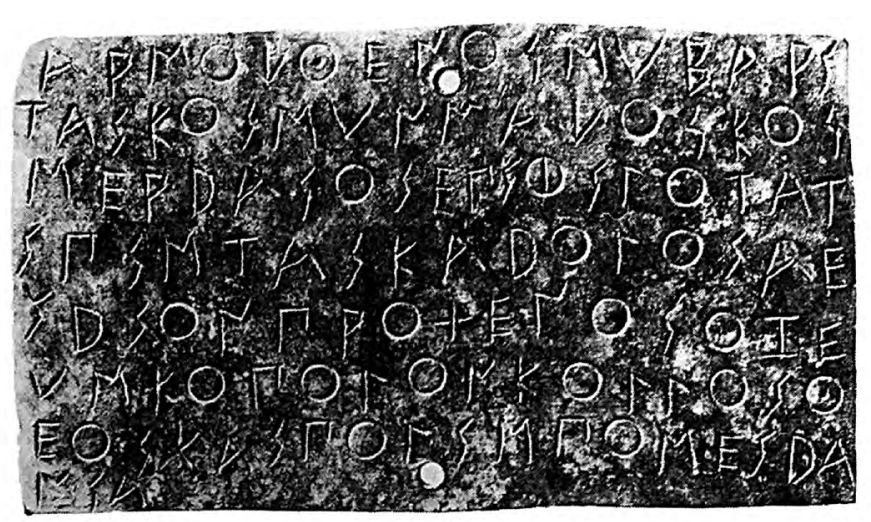

135 SEG 22, 336. Si tratta di un testo di elevato rilievo sul piano storico e testuale, dati

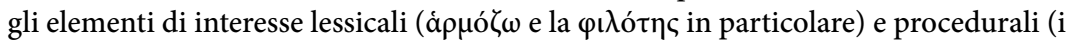
testimoni divini e il ruolo di Poseidonia) che esso presenta. Vid. Meiggs e Lewis 1969, 18-19, nr. 10; Giangiulio 1992; Lombardo 2008, per il quale il documento è posteriore al 510 a.C. 
Il netto incremento di documenti epigrafici che la seconda metà dell'VIII secolo a.C. fa registrare rispetto alla prima metà dello stesso secolo può essere verosimilmente interpretato come il segno dellormai avvenuto successo dell'alfabeto, in tale periodo in diversi e distanti luoghi del mondo greco, sebbene adoperato in maniera ancora poco diversificata in riferimento a usi e scopi. Potremmo verosimilmente essere di fronte, collocando preventivamente l'attività degli innovators e degli early adopters nel periodo compreso fra la metà del IX e la metà dell'VIII sec. a.C., alla fase iniziale del coinvolgimento della early majority degli utenti. Lo sviluppo precoce degli alfabeti regionali, ${ }^{136}$ documentati già per la seconda metà dell'VIII secolo a.C., ${ }^{137}$ sta ad indicare che la scrittura si diffonde nel mondo greco in una maniera decentralizzata, ${ }^{138}$ che lascia ampio spazio agli utenti per modifiche e adattamenti della forma e della sequenza dei grafemi e della corrispondenza fra questi e i fonemi, il che rende più semplice ed efficace l'uso dello strumento in funzione di esigenze specifiche.

Allo stato attuale, per assenza di documentazione, non siamo in grado di precisare il profilo sociale degli innovatori e il motivo che li ha spinti ad agire. Comè noto, l'attenzione è stata concentrata, in passato, su mercanti e aedi, che peraltro possono detenere, in quanto figure spesso caratterizzate da una elevata mobilità, anche su scala mediterranea, ${ }^{139}$ quel carattere 'cosmopolita', in termini di relazioni sociali, che, secondo Rogers contraddistingue generalmente la categoria degli innovators; ${ }^{140}$ tuttavia tanto la già citata teoria dell'origine 'commerciale' quanto quella dell'origine 'aedica' dell'alfabeto ${ }^{141}$ prestano il fianco ad alcune non secondarie perplessità. ${ }^{42}$ Non andrebbe sottovalutato un eventuale ruolo degli artigiani, anch'essi caratterizzati da elevata mobilità ${ }^{143} \mathrm{e}$ presenti, come illustrato, all'interno della documentazione epigrafica di VIII sec. a.C. Di sicuro, come ribadito di recente, ${ }^{144}$ il successo della diffusione di

136 Sul tema vid. le riflessioni di Luraghi 2010 e Johnston 2012.

137 Il lavoro di riferimento sul tema è ancora Jeffery 1990.

138 Su questa modalità della diffusione, di tipo "client-controlled" vid. Rogers 1983, 160-161, 333-346.

139 Vid. Gras 1997, 153-191; Montiglio 2005, 91-117.

140 Rogers 1983, 248.

141 Powell 1989; 1991.

142 Vid. rispettivamente Lombardo 1984 e Cassio 2002.

143 Burkert 1992, 9-40.

144 Henrich 2001, 1009-1010. 
un'innovazione dipende dallo status sociale degli early adopters, fra i quali è necessario che figurino elementi di riconosciuto ed elevato prestigio, il cui comportamento è preso a modello e imitato dagli altri.

\section{$\begin{array}{llllllllllll}\mathbf{B} & \mathbf{I} & \mathbf{B} & \mathbf{L} & \mathbf{I} & \mathbf{O} & \mathbf{G} & \mathbf{R} & \mathbf{A} & \mathbf{F} & \mathbf{I} & \mathbf{A}\end{array}$}

Amadasi Guzzo 1987: M. G. Amadasi Guzzo, "Iscrizioni semitiche di nord-ovest in contesti greci ed italici (X-VII sec. a.C.)”, Dialoghi d'Archeologia 5, 1987, 13-27.

Ampolo 1997: C. Ampolo, "L'interpretazione storica della più antica iscrizione del Lazio (dalla necropoli di Osteria dell'Osa, tomba 482", in: G. Bartoloni (ed.), Le necropoli arcaiche di Veio: giornata di studio in memoria di Massimo Pallottino, Roma 1997, 211-217.

Ampolo 2017: C. Ampolo, "Demarato di Corinto 'bacchiade' tra Grecia, Etruria e Roma: rappresentazione e realtà fonti, funzione dei racconti, integrazione di genti e culture, mobilità sociale arcaica", in: S. Struffolino (ed.), Scritti per il decimo anniversario di Aristonothos [Aristonothos 13/2], Milano 2017, 25-134.

Antonetti, De Vido e Drago 2013: C. Antonetti, S. De Vido e L. Drago, "Lithoi, semata, anathemata. Connotare lo spazio sacro: contesti esemplari tra Grecia ed Etruria”, in: A. Inglese (ed.), Epigrammata 2. Definire, descrivere, proteggere lo spazio sacro. In ricordo di André Laronde, Atti del Convegno di Roma, Roma, 26-27 ottobre 2012, Roma 2013, 1-37.

Arena 1989: R. Arena, "La documentazione epigrafica antica delle colonie greche della Magna Grecia", AnnPisa, serie 3, 19/1, 1989, 15-48.

Arena 1994: R. Arena, Iscrizioni greche arcaiche di Sicilia e Magna Grecia, 3. Iscrizioni delle colonie euboiche, Milano 1994.

Assman 2011: J. Assman, Cultural Memory and Early Civilization. Writing, Remembrance and Political Imagination, Cambridge 2011 [= J. Assman, Das kulturelle Gedächtnis: Schrift, Erinnerung und politische Identität in frühen Hochkulturen, München 1992].

Bagnasco Gianni 2008: G. Bagnasco Gianni, "Comunicare per immagini: una questione di alfabeto", Aristonothos 3, 2008, 47-72.

Bartlett et alii 2011: L. Bartlett, D. López, L. Vasudevan e D. Warriner, “The Anthropology of Literacy”, in: B. A. U. Levison e M. Pollock (eds.), A Companion to the Anthropology of Education, Malden-Oxford 2011, 155-176.

Bartoloni e Nizzo 2005: G. Bartoloni e V. Nizzo, "Lazio protostorico e mondo greco: considerazioni sulla cronologia relativa e assoluta della terza fase laziale", in: G. Bartoloni e F. Delpino (ed.), Oriente e Occidente: metodi e discipline a confronto. Riflessioni sulla cronologia dell'Età del Ferro in Italia. Atti dell'Incontro di studi (Roma, 30-31 ottobre 2003), Pisa-Roma 2005, 409-430.

Barton e Papen 2010: D. Barton e U. Papen, "What Is the Anthropology of Writing?", in: D. Barton e U. Papen (eds.), The Anthropology of Writing. Understanding TextualityMediated Worlds, London-New York 2010.

Bartoněk 1994: A. Bartoněk, "Le iscrizioni greche più arcaiche di Ischia", SborBrno 43, 1994, 113-117. 
Bellelli 2010: V. Bellelli, "L'impatto del mito greco nell'Etruria orientalizzante: la documentazione ceramica", Bollettino di archeologia on-line 1, 2010.

Bellelli 2012: V. Bellelli, "Caere e il mondo greco. Appunti di archeologia e storia”, IncAnt 10, 2012, 137-166.

Bellelli e Benelli 2018: V. Bellelli e E. Benelli, Gli Etruschi. La scrittura, la lingua, la società, Roma 2018.

Benelli 2004: E. Benelli, “Alfabeti greci e alfabeti etruschi”, AnnFaina 9, 2004, 291-305.

Benelli 2007: E. Benelli, Iscrizioni etrusche. Leggerle e capirle, Ancona 2007.

Benelli 2014: E. Benelli, "Etruria, terra di migranti. Mobilità e integrazione di elementi allogeni dalla documentazione epigrafica", in: S. Marchesini, N. Martinelli, M. C. Rossi e A. Paini (ed.), Seconda e terza generazione. Integrazione e identità nei figli di immigrati e coppie miste, Verona 2014, 25-32.

Benzi 1966: M. Benzi, “Gli avori della Marsiliana d'Albegna”, RendLinc 8/21, 1966, 253-292.

Bessios, Tzifopoulos e Kotsonas 2012: M. Bessios, Y. Tzifopoulos e A. Kotsonas, ME $\Theta \Omega N H$

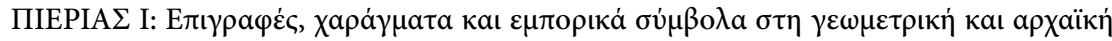

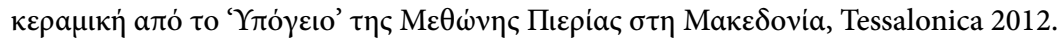

Bianco 2018: S. Bianco, "Herakles nel mondo italico. L'iconografia della fatica degli uccelli stinfalidi sulla ceramica enotria e messapica", in: G. Tagliamonte e M. Spedicato (a cura di), L'inesauribile curiosità. Studi in memoria di Gianni Carluccio, Lecce 2018 81-105.

Bietti Sestieri 1992a: A. M. Bietti Sestieri (a cura di), La necropoli laziale di Osteria dell'Osa, Roma 1992.

Bietti Sestieri 1992b: A. M. Bietti Sestieri, The Iron Age community of Osteria dell'Osa: a study of socio-political development in central Tyrrhenian Italy, Cambridge 1992.

Binek 2017: N. M. Binek, “The Dipylon Oinochoe Graffito. Text or Decoration?”, Hesperia 86/3, 2017, 423-442.

Biondi 1998: L. Biondi, "Recensione a E. Peruzzi, Civiltà greca nel Lazio preromano, Firenze 1998”, PP 53, 1998, 388-400.

Boffa 2015: G. Boffa, "Il vaso ben levigato. Una proposta di lettura per l'iscrizione più antica dalla necropoli di Osteria dell'Osa”, PP 70, 2015, 153-189.

Boffa 2016: G. Boffa, “"Prima colonizzazione” e "primo alfabeto". Il ruolo della scrittura nell'interazione culturale fra le più antiche fondazioni greche in Occidente e l'ambiente italico", in: L. Donnellan, V. Nizzo e G.-J. Burgers (eds), Context of Early Colonization. Acts of the conference "Contextualizing Early Colonization. Archaeology, Sources, Chronology and and Interpretative Models", 1, Roma 2016, 335-349.

Boffa 2017: G. Boffa, "Alfabetari e insegnamento della scrittura in area peuceta fra V e IV secolo a.C.", Historiká. Studi di storia greca e romana 7, 2017, 295-318.

Boffa c. s.: G. Boffa, "Why to write on ceramics. The social implications of writing on ceramic artifacts in early archaic Greece ( $8^{\text {th }}-7^{\text {th }}$ centuries BC)", in: W. Broekaert, A. Delattre, E. Dupraz, M. J. Estarán (eds.), Epigraphy on Ceramics. The Instrumentum domesticum, its Textual Genres and its Functions in Ancient Societies, c. s.

Bonaudo 2008-2009: R. Bonaudo, "In rotta per l'Etruria: Aristonothos, l'artigiano e la metis di Ulisse", AIONArch 15-16, 2008-2009, 143-149.

Bourogiannis 2015: G. Bourogiannis, "Instances of Semitic writing from Geometric and Archaic Greek contexts: an unintelligible way to literacy?", in: G. Garbati e T. Pedrazzi (eds.), Transformations and crisis in the Mediterranean: 'identity' and interculturality in the Levant and Phoenician west during the 12th-8th centuries BCE, Pisa-Roma 2015, 159-170. 
Bowman e Thomas 1983: A. Bowman e D. Thomas, Vindolanda: the Latin writing tablets, London, 1983.

Bowman e Thomas 1994: A. Bowman e D. Thomas, The Vindolanda Writing Tablets, London, 1994.

Braun 1982: T. F. R. G. Braun, “The Greeks in the Near East”, in: Cambridge Ancient History 2, vol. III, Cambridge 1982, 1-31.

Bron e Lemaire 1989: F. Bron e A. Lemaire, "Les inscriptions araméennes de Hazaël", RAssyr $183,1989,35-44$.

Buchner 1966: G. Buchner, "Relazioni tra la necropoli greca di Pitecusa (isola d'Ischia) e la civiltà italica ed etrusca dell'VIII secolo", in: Atti del VI Congresso Internazionale delle Scienze Preistoriche e Protostoriche, Roma 1966, 7-11.

Buchner 1981: G. Buchner, "Pithekoussai: alcuni aspetti peculiari”, ASAtene 59, 1981, 263-273.

Buchner e Bartoněk 1995: G. Buchner, A. Bartoněk, "Die ältesten griechischen Inschriften von Pithekoussai (2. Hälfte des VIII. Bis 1. Hälfte des VI. Jh.)”, Die Sprache 37/2, 1995, 129-237.

Burkert 1992: W. Burkert, The Orientalizing Revolution. Near Eastern Influence on Greek Culture in the Early Archaic Age, Cambridge (MA)-London 1992.

Calce 2011: R. Calce, Graikoi ed Hellenes: storia di due etnonimi, Pisa 2011.

Canali De Rossi 2005: F. Canali De Rossi, Le relazioni diplomatiche di Roma, Vol. I: dalletà regia alla conquista del primato in Italia (753-265 a. C.), Roma 2005.

Cardin 2017: M. Cardin, Oinochoe del Dipylon, AXON, 1/1, 2017, 19-30.

Cardona 1981: G. R. Cardona, Antropologia della scrittura, Torino 1981.

Cassio 1991-1993: A. C. Cassio, "La più antica iscrizione greca di Cuma e $\tau ا(v) v \mu \alpha \iota$ in Omero", Die Sprache, 35, 2, 1991-1993, 187-207.

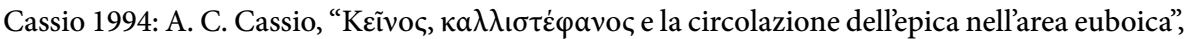
in: B. d'Agostino e D. Ridgway (a cura di), AПОІКІA. Scritti in onore di Giorgio Buchner, Napoli 1994, 55-67.

Cassio 1998: A. C. Cassio, "La cultura euboica e lo sviluppo dell'epica greca", in: M. Bats e B. d'Agostino (a cura di), Euboica: l'Eubea e la presenza euboica in Calcidica e in Occidente. Atti del convegno internazionale di Napoli 13-16 novembre 1996, Napoli 1998, 11-22.

Cassio 1999: A. C. Cassio, "Epica greca e scrittura tra VIII e VII secolo a. C.: madrepatria e colonie d'Occidente", in: G. Bagnasco Gianni e F. Cordano (a cura di), Scritture mediterranee tra il IX e il VII secolo a. C., Milano 1999, 67-84.

Cassio 2002: A. C. Cassio, "Early Editions of the Greek Epics and Homeric Textual Criticism in the Sixth and Fifth Centuries BC", in: F. Montanari e P. Ascheri (a cura di), Omero tremila anni dopo. Atti del Congresso, 6-8 luglio 2000, Roma 2002, 105-136.

Catalin 2016: P. Catalin, "Art and the Alphabet in the Times of the Dipylon Master", Gaia 19, 2016, 25-56.

Coldstream 1993: J. N. Coldstream, "Mixed Marriages at the Frontiers of the Early Greek World”, OxfJA 12, 1993, 89-107.

Colonna 1970: G. Colonna, "Una nuova iscrizione etrusca del VII secolo e appunti sullepigrafia ceretana dell'epoca”, MEFRA 82/2, 1970, 637-672.

Colonna 1995: G. Colonna, "Etruschi a Pitecusa nell'Orientalizzante antico", in: A. Storchi Marino (a cura di), L'incidenza dellantico. Studi in memoria di Ettore Lepore I, Napoli $1995,325-342$.

Colonna 2002: G. Colonna, "Gli Etruschi nel Tirreno Meridionale: tra Mitistoria, Storia e Archeologia”, SE 9/1, 2002,191-206. 
Giovanni Boffa

Colonna 2003: G. Colonna, "Veii", SE 69, 2003, 379-382.

Colonna 2005: G. Colonna, Intervento, in: G. Bartoloni e F. Delpino (a cura di), Oriente e Occidente: metodi e discipline a confronto. Riflessioni sulla cronologia dell'Età del Ferro in Italia, Atti dell'Incontro di studi (Roma, 30 - 31 ottobre 2003), Pisa-Roma 2005, 478-483.

Coulmas 2002: F. Coulmas, Writing Systems. An Introduction to their Linguistic Analysis, Cambridge 2002.

Coulmas 2013: F. Coulmas, Writing and Society. An Introduction, Cambridge 2013.

Cristofani 1971: M. Cristofani, "Per una nuova lettura della pisside della Pania", SE 39, 1971, 63-89.

D’Agostino 1985: B. d'Agostino, "I paesi di provenienza dei coloni e le loro relazioni con il Mediterraneo occidentale”, in: G. Pugliese Carratelli (ed.), Magna Grecia I, Milano 1985, 209-244.

D’Agostino 1994: B. d’Agostino, "La Campania e gli Etruschi”, in: Magna Grecia, Etruschi, Fenici. Atti del trentatreesimo Convegno di Studi sulla Magna Grecia, Taranto, 8-13 ottobre 1993, Taranto 1994, 431-448.

D'Agostino 1999: B. d'Agostino, "Euboean Colonisation in the Gulf of Naples", in: G.R. Tsetskhladze (ed.), Ancient Greeks West and East, Leiden-Boston, 1999, 207-227.

D’Agostino 2003: B. d'Agostino, "Scrittura ed artigianato sulla rotta per l'Occidente", in: S. Marchesini e P. Poccetti (a cura di), Linguistica è storia. Scritti in onore di Carlo De Simone, Pisa 2003, 75-84.

D’Agostino 2008: B. d’Agostino, "Pithecusae e Cuma all'alba della colonizzazione”, in: Atti del XLVIII Convegno di Studi sulla Magna Grecia, Taranto 2008, 171-196.

D’Agostino 2006: B. d'Agostino, “The First Greek in Italy”, in: G. R. Tsetskhladze (ed.), Greek Colonisation. An Account of Greek Colonies and Other Settlements Overseas, 1, LeidenBoston, 2006, 201-237.

D’Agostino e D'Acunto 2008: B. d'Agostino e M. D’Acunto, "La città e le mura: nuovi dati dall'area Nord della città antica", in: Atti del XLVIII Convegno di Studi sulla Magna Grecia, Taranto 2008, 483-522.

De Biasi 2002: R. de Biasi, Che cosê la sociologia della cultura, Roma 2002.

De Kerckhove 2008: D. De Kerckhove, Dall'alfabeto a internet. L'homme "littéré": alfabetizzazione, cultura, tecnologia, Milano-Udine 2008.

Dell'Oro 2017: F. Dell'Oro, "Alphabets and Dialects in the Euboean Colonies of Sicily and Magna Graecia or What Could Have Happened in Methone", in: J. Strauss Clay, I. Malkin e Y. Z. Tzifopoulos (eds.), Panhellenes at Methone. Graphê in Late Geometric and Protoarchaic Methone, Macedonia (ca. 700 BCE), Berlin-Boston 2017, 165-181.

Delpino 1997: F. Delpino, "I Greci in Etruria prima della colonizzazione euboica: ancora su crateri, vite, vino e pennati nell'Italia centrale protostorica”, in: G. Bartoloni (ed.), Le necropoli arcaiche di Veio: giornata di studio in memoria di Massimo Pallottino, Roma 1997, 185-194.

De Rossi 2017: M. I. de Rossi, “Legge di Drero", Axon 1/2, 2017, 7-14.

Dillon 2013: M. P. J. Dillon, “Engendering the Scroll: Girls' and Woman's Literacy in Classical Greece", in: J. E. Grubbs, T. Parkin e R. Bell (eds.), The Oxford Handbook of Childhood and Education in the Classical World, Oxford 2013, 396-417.

Di Tillio 1969: Z. Di Tillio, "Confronti formulari e lessicali tra le iscrizioni esametriche ed elegiache dal VII al V sec. a.C. e l'epos arcaico, I. Iscrizioni sepolcrali”, QuadUrbin 7, 1969, 45-73. 
Donnellan 2016a: L. Donnellan, “A networked view on 'Euboean' colonization”, in: L. Donnellan, V. Nizzo e G.-J. Burgers (eds.), Conceptualizing early Colonisation, Bruxelles-Roma 2016, 149-166.

Donnellan 2016b: L. Donnellan, “'Greek colonisation' and Mediterranean networks: patterns of mobility and interaction at Pithekoussai", Journal of Greek Archaeology 1, 2016, 109-148.

D’Onofrio 1984: A. M. D’Onofrio, “Ancora sulla kylix della T. 2706 di Pontecagnano”, AIONArch 6, 1984, 281-283.

Dubois 1995: L. Dubois, Inscriptions grecques dialectales de Grande Grèce I. Colonies eubéennes. Colonies ioniennes. Emporia, Genève 1995.

Dubois 2002: L. Dubois, Inscriptions grecques dialectales de Grande Grèce, II. Colonies achéennes, Genève 2002.

Duhoux 1984: Y. Duhoux, “Du neuf sur l'ex-voto de Nicomaque (IG XIV 652)”, ZPE 54, 1984, 127-131.

Facchetti 2009: G. M. Facchetti, Antropologia della scrittura, Novara 2009.

Fantalkin 2006: A. Fantalkin, "Identity in the Making. Greeks in the Eastern Mediterranean during the Iron Age", in: A. Villing e U. Schlotzhauer (eds.), Naukratis. Greek diversity in Egypt. Studies on East Greek pottery and exchange in the eastern Mediterranean, London 2006, 199-208.

Fantalkin, Finkelstein e Piasetzky 2011: A. Fantalkin, I. Finkelstein, E. Piasetzky, "Iron Age Mediterranean Chronology: a Rejoinder", Radiocarbon, 53, 1, 2011, 179-198.

Faraone 1996: C. Faraone, “Taking the "Nestor’s Cup Inscription” Seriously: Erotic magic and Conditional Curses in the Earliest Inscribed Hexameters", Classical Antiquity 15/1, 1996, 77-84.

Ferrandini Troisi 2015: F. Ferrandini Troisi, Iscrizioni greche d'Italia. Puglia, Roma 2015.

Finnegan 1988: R. Finnegan, Literacy and Orality, Oxford 1988.

Fraser e Matthews 1997: P. M. Fraser e E. Matthews (eds.), A Lexicon of Greek Personal Names, III.A: the Peloponnese, Western Greece, Sicily, and Magna Graecia, Oxford 1997.

Frisone 2001: F. Frisone, "San Mauro Forte", in: Bibliografia topografica della colonizzazione greca in Italia e nelle isole tirreniche XVIII, Pisa-Roma-Napoli 2001, 79-86.

Gallavotti 1979: C. Gallavotti, Metri e ritmi nelle iscrizioni greche [Bollettino dei classici, Accademia Nazionale dei Lincei, suppl. 2], Roma 1979.

Garbini 1978: G. Garbini, “Iscrizione aramaica a Pitekoussai in 8 sec.", PP 33, 1978, 145-150.

Gentili e Giannini 1977: B. Gentili e P. Giannini, "Preistoria e formazione dellesametro", QuadUrb 26, 1977, 9-51.

Gentili 1967: B. Gentili, "Epigramma ed elegia”, in: Lépigramme grecque. Entretiens sur l'Antiquité classique de la Fondation Hardt, XIV, Vandoeuvres-Genève 1967, 37-90.

Giacometti 2005: D. Giacometti, Metaponto. Gli dei e gli eroi nella storia di una polis di Magna Grecia, Cosenza 2005.

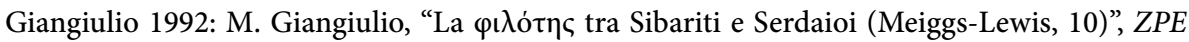
93, 1992, 31-44.

Giangiulio 1993: M. Giangiulio, "La dedica a Eracle di Nicomaco (IG XIV 652). Un'iscrizione arcaica di Lucania ed i rapporti tra Greci ed indigeni nell'entroterra di Metaponto", in: A. Mastrocinque (ed.), Ercole in Occidente, Trento 1993, 29-48.

Giannotta 1985: M. T. Giannotta, "Botromagno", in: Bibliografia topografica della colonizzazione greca in Italia e nelle isole tirreniche, IV, Pisa-Roma 1985, 140-146. 
Ghinatti 2004-2005: F. Ghinatti, “Problemi di epigrafia greca. Gli alfabetari”, MinEpigrP 9-10, 2004-2005, 11-68.

Goody 1986: J. Goody, The Logic of Writing and the Organization of Society, Cambridge 1986.

Goody e Watt 1968: J. Goody e I. Watt, “The Consequences of Literacy”, in: J. Goody (ed.), Literacy in Traditional Societies, Cambridge 1968, 27-68.

Greenhalgh et alii 2005: T. Greenhalgh, G. Robert, F. Macfarlane, P. Bate, O. Kyriakidou e R. Peacock, "Storylines of Research in Diffusion of Innovation: A Meta-narrative Approach to Systematic Review", Social Science \& Medicine, 61/2, 2005, 417-430.

Griswold 2005: W. Griswold, Sociologia della cultura, Bologna 2005.

Guarducci 1967: M. Guarducci, Epigrafia greca, I. Caratteri e storia della disciplina. La scrittura greca dalle origini alletà imperiale, Roma 1967.

Guarducci 1969: M. Guarducci, Epigrafia greca, II. Epigrafi di carattere pubblico, Roma 1969.

Guarducci 1974: M. Guarducci, Epigrafia greca, III. Epigrafi di carattere privato, Roma 1974.

Hall 2004: J. Hall, “How 'Greek' were the early western Greeks?”, in: K. Lomas (ed.), Greek Identity in the Western Mediterranean. Papers in honour of Brian Shefton, Leiden 2004, 35-54.

Hansen 1983: P. A. Hansen, Carmina epigraphica graeca saeculorum VIII-V a. Chr. n., I, Berlin 1983.

Hansen 1985: P. A. Hansen, "The Potter Nicomachus and his dedication (IG 14, $652=C E G$ 396)", ZPE 58, 1985, 231-233.

Hansen 1984: P. A. Hansen, "Recensione a C. Gallavotti, Metri e ritmi nelle iscrizioni greche, Roma 1979”, Classical Antiquity 34/2, 1984, 286-289.

Havelock 1963: E. A. Havelock, Preface to Plato, Cambridge Mass.-London 1963.

Havelock 1982: E. A. Havelock, The Literate Revolution and Its Cultural Consequences, Princeton 1982.

Havelock 1986: E. A. Havelock, The Muse learns to Write. Reflections on Orality and Literacy from Antiquity to the Present, New Haven-London 1986.

Henrich 2001: J. Henrich, "Cultural Transmission and the Diffusion of Innovations: Adoption Dynamics Indicate That Biased Cultural Transmission Is the Predominate Force in Behavioral Change", American Anthropologist 103/4, 2001, 992-1013.

Heubeck 1979: A. Heubeck, Schrift, Göttingen, 1979.

Immerwahr 1990: H. R. Immerwahr, Attic Script. A Survey, Oxford 1990.

Innis 1986: H. A. Innis, Empire and Communications, Toronto 1986.

Janko 2017: R. Janko, "From Gabii and Gordion to Eretria and Methone: the Rise of the Greek Alphabet", in: J. Strauss Clay, I. Malkin e Y. Z. Tzifopoulos (eds.), Panhellenes at Methone. Graphê in Late Geometric and Protoarchaic Methone, Macedonia (ca. 700 BCE), BerlinBoston 2017, 135-164.

Jeffery 1990: L. Jeffery, The Local Scripts of Archaic Greece. A Study of the Origin of the Greek Alphabet and its Development from the Eighth to the Fifth centuries, Oxford 19902.

Johnson 2015: A. W. Johnson, "Learning to Read and Write", in: M. Bloomer (ed.), A Companion to Ancient Education, Malden-Oxford 2015, 137-148.

Johnston 2012: A. W. Johnston, “The life and death of Greek local scripts; not so long durée?”, in: G. van Heems e L. Haumesser (ed.), Régler l'usage: norme et standard dans l'Italie préromaine. Premier atelier: langages. Actes de les Rencontres, Rome 2009, Roma 2012, 319-329.

Johnston e Andriomenou 1989: A. W. Johnston e A. K. Andriomenou, "A Geometric Graffito from Eretria”, $B S A$ 84, 1989, 217-220. 
Jucker 1969: H. Jucker, “Ein protokorinthischer Becher mit etruskischer Inschrift”, SE 37, 1969, 601-605.

Kajava 2010: M. Kajava, ““'Hera non permette che...” Ancora sul dischetto bronzeo di Cuma”, in: L. Chioffi (ed.), Il Mediterraneo e la storia. Epigrafia e archeologia in Campania: letture storiche. Istituto Italiano per gli Studi Filosofici, Napoli, 4-5 dicembre 2008, Napoli 2010, 7-22.

Kenzelmann Pfyffer, Theurillat e Verdan 2005: A. Kenzelmann Pfyffer, T. Theurillat e S. Verdan, "Graffiti d'époque géometrique provenant du sanctuaire d'Apollon Daphnéphoros à Erétrie", ZPE 151, 2005, 51-83.

Lejeune 1983: M. Lejeune, “Sur les abécédaires grecs archaïques”, RPhil 58/1, 1983, 7-12.

Lombardo 1987: M. Lombardo, "Marchands, transactions économiques, écriture", in: M. Detienne (ed.), L'écriture et ses nouveaux objects intéllectuels dans le monde grec, Actes de la Table ronde de Paris, septembre 1984, Paris-Lille, 1987, 159-187.

Lombardo 1999: M. Lombardo, "Vino, poesia e simposio a Metaponto: a proposito di un nuovo documento epigrafico", in: Il vino di Dioniso. Dei e uomini a banchetto in Basilicata. Catalogo della Mostra, Roma 1999, 33-36.

Lombardo 2008: M. Lombardo, "Il trattato tra i Sibariti e i Serdaioi: problemi di cronologia e di inquadramento storico", StAnt 12, 2008, 49-60.

Lombardo e Frisone 2011: M. Lombardo, F. Frisone, "Vino e società nelle città magnogreche: le tradizioni letterarie e i documenti epigrafici", in: La vigna di Dioniso. Vite, vino e culti in Magna Grecia. Atti del XLIX Convegno di Studi sulla Magna Grecia, Taranto 24-28 settembre 2009, Taranto 2011, 283-347.

Luraghi 2010: N. Luraghi, “The Local Scripts from Nature to Culture”, Classical Antiquity 29, 2010, 68-91.

Maggiani 2009: A. Maggiani, "Rivista di epigrafia etrusca (Ager Volaterranus)", SE 73, 2009, 371-375.

Maras 2011: D. F. Maras, "Ai confini delloralità. Le forme e i documenti del dono nelle aristocrazie orientalizzanti etrusche", in: V. Nizzo (a cura di), Dalla nascita alla morte: antropologia e archeologia a confronto. Atti dell'Incontro Internazionale di studi in onore di Claude Lévi-Strauss, Roma, Museo Nazionale preistorico etnografico "Luigi Pigorini" 21 maggio 2010, Roma 2011, 703-713.

Maras 2012a: D. F. Maras, "La scrittura dei principi etruschi”, in: M. Sannibale e A. Mandolesi (a cura di), Etruschi. L'ideale eroico e il vino lucente. Catalogo della mostra (Asti 17 marzo-15 luglio 2012), Milano 2012, 103-109.

Maras 2012b: D. F. Maras, "Interferenza e concorrenza di modelli alfabetici e sistemi scrittori nell'Etruria arcaica”, in: G. van Heems e L. Haumesser (ed.), Régler l'usage: norme et standard dans l'Italie préromaine. Premier atelier: langages. Actes de les Rencontres, Rome 2009 [MEFRA 124/2], 2012, 331-344.

Marchesini 2013: S. Marchesini, "Quali lingue, quali popoli nell'Apulia di V e IV secolo", in: L. Todisco (a cura di), La comunicazione verbale tra Greci e indigeni in Apulia nel V-IV secolo a. C.: quali elementi? Atti del Convegno, Bari 2012, Napoli 2013, 19-33.

Marek 1993: C. Marek, "Euboia und die Entstehung der Alphabetschrift bei den Griechen", Klio 75, 1993, 27-44.

Marrou 1964: H.-I. Marrou, Histoire de léducation dans l'Antiquité, Paris $1964^{6}$.

Martelli 1981: M. Martelli, "Le manifestazioni artistiche", in: M. Cristofani (a cura di), Gli Etruschi in Maremma: popolamento e attività produttive, Milano 1981, 221-284. 
Mazarakis Ainian 2007: A. Mazarakis Ainian, "EN $\triangle$ ON $\Sigma$ KAПTE. The Tale of an Excavation", in: A. Mazarakis Ainian (ed.), Oropos and Euboea in the Early Iron Age. Acts of an International Round Table, University of Thessaly, June 18-20, 2004, Volos 2007, 21-59.

Meiggs e Lewis 1969: R. Meiggs e D. Lewis, A Selection of Greek Historical Inscriptions, Oxford 1969.

Mele 2008: A. Mele, "Cuma in Opicia tra Greci e Romani”, in: Cuma. Atti del XLVIII Convegno di Studi sulla Magna Grecia, Taranto-Cuma, 27 settembre - 1 ottobre 2008, Taranto 2008, 77-167.

Méndez Dosuna 2017: J. Méndez Dosuna, "Methone of Pieria: a Reassessment of Epigraphical Evidence (with a Special Attention to Pleonastic Sigma)", in: J. Strauss Clay, I. Malkin e Y. Z. Tzifopoulos (eds.), Panhellenes at Methone. Graphê in Late Geometric and Protoarchaic Methone, Macedonia (ca. 700 BCE), Berlin-Boston 2017.

Mermati 2012: F. Mermati, "Osservazioni sulla costruzione dell'identità coloniale tra Pithekoussai e Cuma”, in J.-P. Descoeudres e A. S. Paspalas, Zagora in Context: Settlements and Intercommunal Links in the Geometric Period (900-700 BC). Proceedings of the conference held by The Australian Archaeological Institute at Athens and The Archaeological Society at Athens. Athens, 20-22 May, Sydney 2012, 283-307.

Mermati 2014: F. Mermati, "Ibridismo materiale e ibridismo culturale. La produzione ceramica pitecusano-cumana a contatto con l'altro" tra la metà dell'VIII sec. e la prima metà del VII sec. a.C.", in: J. M. Álvarez, T. Nogales e I. Rodà (eds.), Centro y periferia en el mundo clásico. S. 5. Griegos en el Mediterráneo. Centros, factorías y comercio, Actas XVIII Congreso Internacional Arqueología Clásica, Mérida 2014, 575-578.

Milne 1945: M. J. Milne, “A Prize for Wool-Working”, AJA 49, 1945, 528-535.

Minto 1921: A. Minto, Marsiliana d'Albegna. Le scoperte archeologiche del principe don Tommaso Corsini, Firenze 1921.

Montiglio 2005: S. Montiglio, Wandering in Ancient Greek Culture, Chicago-London 2005.

Moranti 1972: M. Moranti, "Formule metriche nelle iscrizioni greche arcaiche", QuadUrb 13, 1972, 7-23.

Murray 1994: O. Murray, "Nestor's cup and the Origins of Greek Symposion”, AIONArch 1, 1994, 47-54.

Murray 2009: O. Murray, “The Culture of the Symposion”, in: K. A. Raaflaub e H. van Wees, (eds.), A Companion to Archaic Greece, Oxford 2009, 508-523.

Murray 2011: O. Murray, "Il simposio tra Oriente e Occidente", in: La vigna di Dioniso. Vite, vino e culti in Magna Grecia. Atti del XLIX Convegno di Studi sulla Magna Grecia, Taranto 24-28 settembre 2009, Taranto 2011, 55-69.

Nafissi 1998: M. Nafissi, “Xainein: le gambe di Melosa (LSAG2, 283 nr. 1)”, PP 1998, 30-39.

Neeft 1987: C.W. Neeft, Protokorinthian Subgeometric Aryballoi, Amsterdam 1987.

Nijboer e Van Der Plicht 2008: A. J. Nijboer e J. Van Der Plicht, “The Iron Age in the Mediterranean: Recent Radiocarbon Research at the University of Groningen", in: D. Brandherm e M. Trachsel (eds.), A New Dawn for the Dark Age? Shifting Paradigms in Mediterranean Iron Age Chronology. Proceedings of the XV World Congress (Lisbon, 4-9 September 2006), Oxford 2008, 103-118.

Oikonomaki 2017: N. Oikonomaki, "Local 'Literacies' in the Making: Early Alphabetic Writing and Modern Literacy Theories", in: J. Strauss Clay, I. Malkin e Y. Z. Tzifopoulos (eds.), Panhellenes at Methone. Graphê in Late Geometric and Protoarchaic Methone, Macedonia (ca. 700 BCE), Berlin-Boston 2017, 261-284. 
Olson e Torrance 1991: D. R. Olson e N. G. Torrance (eds.), Literacy and orality, Cambridge 1991.

Olson e Cole 2006: D. R. Olson e M. Cole, Technology, Literacy, and the Evolution of Society. Implications of the Work of Jack Goody, Mahwah-London 2006.

Olson e Torrance 2001: D. R. Olson e N. G. Torrance, (eds.), The Making of Literate Societies, Oxford 2001.

Olson e Torrance 2009: D. R. Olson e N. G. Torrance, Cambridge Handbook of Literacy, Cambridge 2009.

Olson, Torrance e Hildyard 1985: D. R. Olson, N. G. Torrance e A. Hildyard (eds.), Literacy, language and learning: The nature and consequences of reading and writing, Cambridge 1985.

Ong 2002: W. J. Ong, Orality and Literacy. The Technologizing of the Word, London-New York $2002^{2}$.

Osborne e Pappas 2007: R. Osborne e A. Pappas, "Writing on Archaic Greek pottery", in: Z. Newby e R. Leader-Newby (eds.) Art and Inscriptions in the Ancient World, Cambridge 2007, 131-155.

Pandolfini e Prosdocimi 1990: M. Pandolfini e A. L. Prosdocimi, Alfabetari ed insegnamento della scrittura in Etruria e nell'Italia antica, Firenze, 1990.

Pappas 2011: A. Pappas, "Arts in letters. The aesthetics of ancient Greek writing”, in: M. Dalbello e M. Shaw (eds.), Visible Writings: Cultures, Forms, Readings, New Brunswick, 2011, 37-54.

Pavese 1996: C. O. Pavese, "La iscrizione sulla kotyle di Nestor da Pithekoussai”, ZPE 114, 1996, $1-23$.

Peruzzi 1997: E. Peruzzi, “Cultura greca Gabii nel secolo VIII”, PP 47, 1997, 459-468.

Peruzzi 1998: E. Peruzzi, Civiltà greca nel Lazio preromano, Roma 1998.

Poccetti 2015: P. Poccetti, “Alphabet grec et langues indigènes de la Grande Grèce entre unité et variété", in: R. Roure (éd.), Contacts et acculturations en Méditerranée occidentale. Hommages à Michel Bats. Actes du colloque de Hyères, 15-18 septembre 2011, Aix-enProvence 2015, 511-523.

Poetto e Facchetti 2009: M. Poetto e G. Facchetti, "Laryballos di Ara日 Numasiana", Oebalus 4, 2009, 365-380.

Popham, Sackett e Themelis 1980: M. R. Popham, L. H. Sackett e P. G. Themelis, Lefkandi I: The Iron Age, London 1980.

Powell 1989: B. B. Powell, "Why Was the Greek Alphabet Invented? The Epigraphical Evidence", CA 8/2, 1989, 321-350.

Powell 1991: B. B. Powell, Homer and the Origin of the Greek Alphabet, Cambridge 1991.

Probert 2015: P. Probert, Early Greek Relative Clauses, Oxford 2015.

Prosdocimi 1999: A. L. Prosdocimi, "Sicilia. Note sullalfabetizzazione", in: M. I. Gulletta (ed.), Sicilia Epigraphica. Atti del convegno internazionale, Erice, 15-18 Ottobre 1998, Pisa 1999, 465-482.

Rebillard 1989: L. Rebillard, "Exékias apprend à écrire: diffusion de l'écriture du Céramique au VIe s. av. J.C.", in: C. Baurain e C. Bonnet (eds.), Phoinikeia Grammata. Lire et écrire en Mediteranée. Actes du colloque de Liège, 15-18 novembre 1989, Namur 1989, 549-564.

Ribezzo 1920: F. Ribezzo, "Le iscrizioni greco-arcaiche di Cuma”, Rivista Indo-Greco-Italica 3/3-4, 1920, 71-87.

Ridgway 1984: D. Ridgway, L’alba della Magna Grecia, Milano 1984.

Ridgway 1996: D. Ridgway, “Greek Letters at Osteria dell'Osa”, OpRom 20, 1996, 87-97. 
Giovanni Boffa

Ridgway 1992: D. Ridgway, The First Western Greeks, Cambridge 1992.

Ridgway 1997: D. Ridgway, "Nestor's Cup and the Etruscans", OxfJA 16, 3, 1997, 325-344.

Ridgway 2009: D. Ridgway, "Eroi segreti della storia preromana d'Italia”, in: J.-P. Brun (éd.), Artisanats antiques d'Italie et de Gaule. Mélanges offerts à Maria Francesca Buonaiuto, Naples 2009, 15-21.

Ridgway 2012: D. Ridgway, "Mobilità mediterranea: traffici e presenze egee e orientali in Occidente tra IX e VIII sec. a.C., in: Alle origini della Magna Grecia. Mobilità, migrazioni, fondazioni. Atti del L Convegno di Studi sulla Magna Grecia, Taranto, 1-4 ottobre 2010, Taranto 2012, 259-269.

Rix 1984: H. Rix, "La scrittura e la lingua", in: M. Cristofani (ed.), Gli Etruschi, una nuova immagine, Firenze 1984.

Rix 1991: H. Rix, Etruskische Texte. Editio minor, I-II, Tübingen 1991.

Rix 2002: H. Rix, Sabellische Texte. Die Texte des Oskischen, Umbrischen und Südpikenischen, Heidelberg 2002.

Robb 1994: K. Robb, Literacy and Paideia in Ancient Greece, Oxford 1994.

Rogers 1983: E. Rogers, Diffusion on Innovations, New York $1983^{3}$.

Ruijgh 1998: C. J. Ruijgh, "Sur la date de la création de l’alphabet grec", Mnemosyne 51, 1998, 658-687.

Saltini Semerari 2016: G. Saltini Semerari, "Greek-Indigenous intermarriage: a gendered perspective”, in: L. Donnellan, V. Nizzo e G.-J. Burgers (eds.), Conceptualising Early Colonisation, Bruxelles-Roma 2016, 77-87.

Santoro 1978: C. Santoro, "La situazione storico-linguistica della peucezia preromana alla luce di nuovi documenti", in C. Santoro e C. Marangio (ed.), Studi storico-linguistici in onore di Francesco Ribezzo, Mesagne 1978, 219-330.

Sciacca 2004: F. Sciacca, "I buccheri della tomba Calabresi: una produzione di prestigio dell'orientalizzante medio ceretano", in: A. Naso (a cura di), Appunti sul bucchero, Atti delle Giornate di Studio, Firenze 2004, 29-42.

Sciolla 2006: L. Sciolla, Sociologia dei processi culturali, Bologna 2006.

Slings 1998: R. S. Slings, "Tsadē and Hēe: Two problems in the early History of the Greek Alphabet", Mnemosyne 51/6, 1998, 641-657

Small 2004: A. Small, "Pots, People, and Places in fourth-century B.C.E. Apulia", in: T.H. Carpenter, K.M. Lynch e E. G. D. Robinson (ed.) The Italic People of Ancient Apulia. New Evidence from Pottery for Workshops, Markets, and Customs, Cambridge 2004, 13-35.

Taylor e Olson 1995: I. Taylor e D. R. Olson, Scripts and Literacy. Reading and Learning to read Alphabets, Syllabaries and Characters, New York 1995.

Theurillat 2007: T. Theurillat, "Early Iron Age Graffiti from the Sanctuary of Apollo at Eretria", in: A. Mazarakis Ainian (ed.), Oropos and Euboea in the Early Iron Age, Acts of an International Round Table (University of Thessaly, June 18-20 2004), Volos 2007, 331-344.

Thomas 1989: R. Thomas, Oral Tradition and Written Record in Classical Athens, Cambridge 1989.

Thomas 1992: R. Thomas, Literacy and Orality in Ancient Greece, Cambridge 1992.

Thomas 2009: R. Thomas, "Writing, Reading, Public and Private "Literacies": Functional Literacy and Democratic Literacy in Greece", in: A. Johnson e H. N. Parker (eds.), Ancient Literacies. The Culture of Reading in Greece and Rome, Oxford 2009, 13-45.

Tramonti 1983: A. Tramonti, Note per la Carta Archeologica di San Mauro Forte, Galatina 1983. Valerio 2017: F. Valerio, “Coppa di Nestore”, Axon 1/1, 2017, 11-17. 
Van der Plicht, Bruins e Nijboer 2009: J. van der Plicht, H. L. Bruins e A. J. Nijboer, “The Iron Age around the Mediterranean: a High Chronology Perspective from the Groningen Radiocarbon Database", Radiocarbon 51/1, 2009, 213-242.

Van Dongen 2008: E. van Dongen, "The study of Near Eastern influences on Greece: Towards the point", Kaskal. Rivista di storia, ambienti e culture del Vicino Oriente Antico, 5, 2008, 233-250.

Von Pape 2009: T. von Pape, "Media Adoption and Diffusion", in: T. Hartmann (ed.), Media Choice. A Theoretical and Empirical Overview, New York 2009, 274-282.

Wachter 2005: R. Wachter, “Annex zu Fragment 3 der Graffiti von Eretria”, ZPE 151, 2005, 84-86.

Wallace 2011: R. Wallace, "The Latin Alphabet and Orthography”, in: J. Clackson (ed.), A Companion to the Latin Language, Malden-Oxford 2011, 9-28.

Wecowski 2014: M. Wecowski, The Rise of the Greek Aristocratic Banquet, Oxford 2014.

Wecowski 2017: M. Wecowski, "Wine and the Early History of the Greek Alphabet. Early Greek Vase-Inscriptions and the Symposion", in: J. Strauss Clay, I. Malkin e Y. Tzifopoulos (eds.), Panhellenes at Methone: Graphê in Late Geometric and Protoarchaic Methone, Macedonia (ca 700 BCE), Berlin-Boston 2017, 309-358.

West 1988: M. L. West, “The Rise of the Greek Epic”, JHS 108, 1988, 151-172.

West 2011: M. L. West, “The Homeric Question Today”, Proceedings of the American Philosophical Society 155/4, 2011, 383-393.

West 2014: M. L. West, The making of the «Odyssey», Oxford 2014.

West 2015: W. C. West, III, "Learning the Alphabet: Abecedaria and the Early Schools in Greece”, GrRomByzSt 55, 2015, 52-71.

Wiseman 1955: D. J. Wiseman, "Assyrian Writing Boards", Iraq 17, 1955, 3-13.

Wolicki 2015: A. Wolicki, "The Education of Women in Ancient Greece”, in: W. M. Bloomer (ed.), A Companion to Ancient Education, Malden-Oxford 2015, 305-320.

Woodard 2017: R. D. Woodard, "Alphabet and Phonology at Methone: Beginning a Typology of

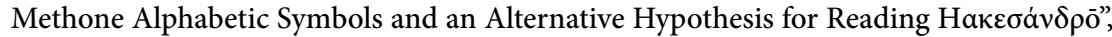
in: J. Strauss Clay, I. Malkin e Y. Z. Tzifopoulos (eds.), Panhellenes at Methone. Graphê in Late Geometric and Protoarchaic Methone, Macedonia (ca. 700 BCE), Berlin-Boston 2017, 182-218.

Woudhuizen 1988-89: F. C. Woudhuizen, "Epigraphic Evidence for Alien residente of a Third Kind at Pithecussae/Cumae, Italy, c. 750-675 B.C.", Talanta 20-21, 1988-89, 97-108.

Ziskowski 2007: A. Ziskowski, "Debating the Origins of Colonial Women in Sicily and South Italy", in: A.-M. Knoblauch, T. Papillon, The Mythology and Iconography of Colonization: a Special Themed Issue of Electronic Antiquity. Electronic Antiquity 11, 1, 2007, 139-157. 